\title{
Exact mode volume and Purcell factor of open optical systems
}

\author{
E. A. Muljarov and W. Langbein \\ School of Physics and Astronomy, Cardiff University, Cardiff CF24 3AA, United Kingdom \\ (Received 22 October 2014; revised manuscript received 29 March 2016; published 27 December 2016)
}

\begin{abstract}
The Purcell factor quantifies the change of the radiative decay of a dipole in an electromagnetic environment relative to free space. Designing this factor is at the heart of photonics technology, striving to develop ever smaller or less lossy optical resonators. The Purcell factor can be expressed using the electromagnetic eigenmodes of the resonators, introducing the notion of a mode volume for each mode. This approach allows an analytic treatment, reducing the Purcell factor and other observables to sums over eigenmode resonances. Calculating the mode volumes requires a correct normalization of the modes. We introduce an exact normalization of modes, not relying on perfectly matched layers. We present an analytic theory of the Purcell effect based on this exact mode normalization and the resulting effective mode volume. We use a homogeneous dielectric sphere in vacuum, which is analytically solvable, to exemplify these findings. We furthermore verify the applicability of the normalization to numerically determined modes of a finite dielectric cylinder.
\end{abstract}

DOI: 10.1103/PhysRevB.94.235438

In his short communication [1] published in 1946, Purcell introduced a factor of enhancement of the spontaneous emission rate of a dipole of frequency $\omega$ resonantly coupled to a mode in an optical resonator, which is now known as the Purcell factor (PF). He estimated this factor as

$$
F=\frac{6 \pi c^{3} Q_{n}}{\omega^{3} V_{n}},
$$

with the speed of light $c$, the quality factor $Q_{n}$ of the optical mode $n$, and its effective volume $V_{n}$, the latter being evaluated as simply the volume of the resonator. This rough estimate of $V_{n}$ has subsequently been refined [2,3] to

$$
\frac{1}{V_{n}}=\left[\mathbf{e} \cdot \mathbf{E}_{n}\left(\mathbf{r}_{d}\right)\right]^{2}
$$

where $\mathbf{r}_{d}$ is the position of the dipole and $\mathbf{e}$ the unit vector of its polarization. In this expression, the electric field of the mode $\mathbf{E}_{n}(\mathbf{r})$ is normalized [2] as

$$
1=\int_{\mathcal{V}} \varepsilon(\mathbf{r}) \mathbf{E}_{n}^{2}(\mathbf{r}) d \mathbf{r}
$$

where $\varepsilon(\mathbf{r})$ is the permittivity of the resonator. The integration is performed over the "quantization volume" $\mathcal{V}$. However, for an open system this volume is not defined, and simply extending $\mathcal{V}$ over the entire space leads to a diverging normalization integral since eigenmodes of an open system grow exponentially outside of the system due to their leakage. This issue was mostly ignored in the literature and patched by phenomenologically choosing a finite integration volume. Such an approach can result in relatively small errors when dealing with modes of high $Q_{n}$, as we will see later. However, the fundamental problem of calculating the exact mode normalization and thus of the mode volume remained.

Recently, a solution to this problem has been suggested. Kristensen et al. [4,5] have used the normalization which was introduced by Leung et al. [6] for one-dimensional (1D) optical systems and later applied [7] to three dimensions. In this approach, the volume integral in Eq. (3) is complemented by a surface term and the limit of infinite volume $\mathcal{V}$ is taken:

$$
1=\lim _{\mathcal{V} \rightarrow \infty} \int_{\mathcal{V}} \varepsilon(\mathbf{r}) \mathbf{E}_{n}^{2}(\mathbf{r}) d \mathbf{r}+\frac{i c}{2 \omega_{n}} \oint_{S_{\mathcal{V}}} \mathbf{E}_{n}^{2}(\mathbf{r}) d S,
$$

where $\omega_{n}$ is the complex eigenfrequency of the mode and $S_{\mathcal{V}}$ is the boundary of $\mathcal{V}$. It was found [4] that for high- $Q$ modes, the surface term was leading to an approximately converging value of the normalization with increasing $\mathcal{V}$, for the limited volume range available in numerical simulations. We show later that Eq. (4), which we call in the following the Leung-Kristensen (LK) normalization, is diverging in the limit $\mathcal{V} \rightarrow \infty$, with a leading term scaling with the radius $R$ of a spherical volume $\mathcal{V}$ as $\exp \left(2 i \omega_{n} R / c\right) / R^{2}$ (see Appendices $\mathrm{C}$ and G).

In an alternative method introduced by Sauvan et al. [8], the mode volume is determined from the mode field calculated using a perfectly matched layer (PML), which is widely used in electromagnetic software packages. A PML is an effective absorbing layer with material properties derived from a coordinate transform from real to complex coordinates. It allows one to effectively provide outgoing boundary conditions within a finite simulation volume. In this way, the divergence of the normalization Eq. (3) is removed by converting the radiative losses to the outside region into absorptive losses within the simulation volume. It has been shown in [8] on a $1 \mathrm{D}$ analytic example that the resulting mode normalization is independent of the PML coordinate transform for frequency-independent (nondispersive) transforms (see also Appendix D).

The normalization of eigenstates is also at the heart of any perturbation theory, and only in 2010 such a theory, the resonant-state expansion (RSE), was formulated [9] and subsequently applied to 1D, 2D, and 3D systems [9-14], showing its ability to accurately and efficiently calculate resonant states (RSs) — the eigenmodes — of a perturbed open optical system, using the spectrum of RSs of a simpler, unperturbed one. The normalization of RSs introduced in [9] is a key element of the RSE.

Here, we present a rigorous theory of the Purcell effect, based on an exact formula for the mode volume in a general finite three-dimensional optical system, described by a local frequency-dependent permittivity. We illustrate this theory on 
the exactly solvable model of a dielectric spherical resonator. We also verify the applicability of the normalization to numerically determined modes of a finite dielectric cylinder for which no analytical solution is known.

In the weak coupling regime, the spontaneous emission rate of a quantum dipole, which is determining the local density of states and the spectral function of the resonator, has the form [15-17]

$$
\gamma(\omega)=-\frac{\omega^{2}}{\varepsilon_{0} \hbar c^{2}} \boldsymbol{\mu} \cdot \operatorname{Im} \hat{\mathbf{G}}\left(\mathbf{r}_{d}, \mathbf{r}_{d} ; \omega\right) \boldsymbol{\mu},
$$

as detailed in Appendix A. Here, $\boldsymbol{\mu}=\mu \mathbf{e}$ is the electric dipole moment and $\varepsilon_{0}$ is the vacuum permittivity. The dyadic Green's function $(\mathrm{GF}) \hat{\mathbf{G}}$ which contributes to Eq. (5) respects the outgoing wave boundary conditions and satisfies Maxwell's wave equation with a delta function source term,

$$
\left(\frac{\omega^{2}}{c^{2}} \hat{\boldsymbol{\epsilon}}(\mathbf{r})-\nabla \times \nabla \times\right) \hat{\mathbf{G}}\left(\mathbf{r}, \mathbf{r}^{\prime} ; \omega\right)=\hat{\mathbf{1}} \delta\left(\mathbf{r}-\mathbf{r}^{\prime}\right),
$$

where $\hat{\boldsymbol{\epsilon}}(\mathbf{r})$ is the dielectric tensor of the open optical system and $\hat{\mathbf{1}}$ is the unit tensor. The permeability is assumed to be $\hat{\boldsymbol{\mu}}(\mathbf{r})=\hat{\mathbf{1}}$ throughout this paper for simplicity. With modern electromagnetic software, Eq. (6) can be solved numerically by replacing the $\delta$-like source term with a finite-size dipole at a given position $\mathbf{r}^{\prime}$. The mode volume can then be evaluated by calculating numerically the EM field emitted at frequencies close to the pole of the GF under consideration using an iterative algorithm converging towards the pole, as has been recently shown [18]. Our approach is instead based on the exact form of the solution of Eq. (6).

In the following we provide exact expressions for the mode normalization, mode volume, and resulting PF, which use only the mode field in a finite volume and its frequency, so that they are applicable for modes calculated by any available means.

Inside the optical system, i.e., within the volume of inhomogeneity of $\hat{\boldsymbol{\epsilon}}(\mathbf{r})$, the GF has the following spectral representation $[9,11,13]$ :

$$
\hat{\mathbf{G}}\left(\mathbf{r}, \mathbf{r}^{\prime} ; \omega\right)=c^{2} \sum_{n} \frac{\mathbf{E}_{n}(\mathbf{r}) \otimes \mathbf{E}_{n}\left(\mathbf{r}^{\prime}\right)}{2 \omega_{n}\left(\omega-\omega_{n}\right)},
$$

in which the sum is taken over all RSs (see also Appendix B). RSs are the optical modes of the system, the eigensolutions of Maxwell's wave equation satisfying the outgoing wave boundary conditions. The eigenfrequency $\omega_{n}=\Omega_{n}-i \Gamma_{n}$ of a RS is generally complex and contains the position $\Omega_{n}$ of the resonance and its half width at half maximum $\Gamma_{n}$. RSs with $\Gamma_{n} \neq 0$ contribute in pairs: Each RS with $\omega_{n}$ and $\mathbf{E}_{n}$ has a counterpart with the eigenfrequency $-\omega_{n}^{*}$ and the wave function $\mathbf{E}_{n}^{*}$. The quality factor of a $\mathrm{RS}$ is given by $Q_{n}=\Omega_{n} / 2 \Gamma_{n}$. The spectral representation Eq. (7) requires that the RSs (with $\omega_{n} \neq 0$ ) are normalized according to

$$
\begin{aligned}
1= & \int_{\mathcal{V}} \mathbf{E}_{n}(\mathbf{r}) \cdot \hat{\boldsymbol{\epsilon}}(\mathbf{r}) \mathbf{E}_{n}(\mathbf{r}) d \mathbf{r} \\
& +\frac{c^{2}}{2 \omega_{n}^{2}} \oint_{S_{\mathcal{V}}}\left[\mathbf{E}_{n} \cdot \frac{\partial}{\partial s}(\mathbf{r} \cdot \nabla) \mathbf{E}_{n}-\frac{\partial \mathbf{E}_{n}}{\partial s} \cdot(\mathbf{r} \cdot \nabla) \mathbf{E}_{n}\right] d S,
\end{aligned}
$$

where the first integral is taken over a simply connected volume $\mathcal{V}$ enclosing the inhomogeneity of the system, while the second integral is taken over the closed surface $S_{\mathcal{V}}$ of the volume $\mathcal{V}$, with the normal derivative $\partial / \partial s=\hat{\mathbf{n}} \cdot \nabla$ using the outward surface normal $\hat{\mathbf{n}}$. Equation (8) is the correct mode normalization, consistent with the spectral representation Eq. (7) of the GF, and is valid for any finite volume $\mathcal{V}$ containing the system - the volume dependencies of both integrals in Eq. (8) exactly compensate each other. It is suited for arbitrary finite open optical systems, and we show examples for exactly solvable spherical systems and for nonspherical system calculated numerically (see Appendix G).

The expression in the surface term of Eq. (8) can be simplified in spherical coordinates to radial derivatives, using $\mathbf{r} \cdot \boldsymbol{\nabla}=r \partial / \partial r$. Furthermore, choosing $\mathcal{V}$ in the form of a finite sphere in 3D or a finite cylinder in 2D yields $\partial / \partial s=\partial / \partial r$ and a simpler form of the normalization of RSs $[9,11,13]$, see Eq. (B.18) of Appendix B. A derivation of the normalization Eq. (8) using a spherical volume $\mathcal{V}$ is given in [13]. Since a convenient normalization volume $\mathcal{V}$ can be different from a sphere, we have generalized here the normalization to an arbitrarily shaped simply connected volume and have presented it in the form independent of the coordinate system used. The related derivation of Eq. (8) is provided in Appendix B. In the presence of a frequency dispersion of the permittivity, which is important, e.g., in metallic resonators, the dielectric constant $\hat{\boldsymbol{\epsilon}}(\mathbf{r})$ in Eq. (8) is replaced by $\partial\left(\omega^{2} \hat{\boldsymbol{\epsilon}}(\mathbf{r}, \omega)\right) / \partial\left(\omega^{2}\right)$, as also shown in Appendix B, and in the case of the dispersion also of the background material (replacing the vacuum treated above), the surface term in Eq. (8) acquires an additional factor $\varepsilon^{-1}(\omega) \partial\left(\omega^{2} \varepsilon(\omega)\right) / \partial\left(\omega^{2}\right)$.

Comparing the LK and the exact normalization, we note that Eq. (4) has an additional prefactor $\omega_{n} / c$, the wave vector, while Eq. (8) calculates explicitly the normal derivatives of the fields, which results in the factor $\omega_{n} / c$ only for fields propagating normal to the surface of integration. This observation clarifies the qualitative difference between the two normalizations: the LK normalization assumes normal propagation, while the exact normalization takes into account the actual propagation direction. The exact normalization in the form of Eq. (8) depends on both first and second spatial derivatives of the fields. However, as shown in Appendix B, one can use Maxwell's equations to convert Eq. (8) into a form containing only first derivatives, see Eq. (B27). This can be advantageous for application to RSs calculated using numerical solvers such as the finite element method (FEM), as shown in Appendix G. We find that Eq. (8) is robust against the choice of grids and PML thickness used in the FEM. Furthermore, since it can be evaluated close to the system, the surface area can be minimized, reducing the numerical error and the required simulation domain. The LK normalization instead not only diverges for large $R$, as shown below, but also has significant errors for small $R$, since the non-normal field propagation results in an error scaling as $R^{-2}$. Therefore, even for RSs of high $Q_{n}$ for which the divergence with $R$ is slow, the LK normalization requires one to use a simulation domain much larger than the system size, which is computationally costly.

The spectral representation Eq. (7) determines the exact PF, taking into account the contribution of all significant modes. Indeed, using Eqs. (5) and (7), the PF in the weak coupling 
regime is obtained as

$$
F(\omega)=\frac{\gamma(\omega)}{\gamma_{0}(\omega)}=\frac{3 \pi c^{3}}{\omega} \sum_{n} \operatorname{Im} \frac{1}{V_{n} \omega_{n}\left(\omega_{n}-\omega\right)},
$$

which requires using the exact mode volume $V_{n}$ given by Eq. (2) with the electric field $\mathbf{E}_{n}(\mathbf{r})$ of the RS respecting the correct normalization Eq. (8).

Here $\gamma_{0}=\omega^{3} \mu^{2} /\left(6 \pi \varepsilon_{0} \hbar c^{3}\right)$ is the radiative decay rate of the dipole in free space [1], which can be deduced from Eq. (5) using the GF of empty space [19], as shown in Appendix A. If a single mode $n$ dominates in Eq. (9), the $\mathrm{PF}$ on resonance $\left(\omega=\operatorname{Re} \omega_{n}\right)$ can be approximated as $F(\omega) \approx 6 \pi c^{3} Q_{n} /\left[\omega^{2} \operatorname{Re}\left(\omega_{n} V_{n}\right)\right]$. For a high- $Q$ mode, the eigenfrequency and mode volume are approximately real, and the latter formula reproduces Purcell's result Eq. (1) when using the correct mode volume $V_{n}$.

For illustration, we have calculated the mode volume and PF of a dielectric spherical resonator of radius $a$ with homogeneous permittivity $(\varepsilon=4)$ surrounded by vacuum, for a point dipole placed at $\left|\mathbf{r}_{d}\right|=0.9 a$ with direction $\mathbf{e}=(0,0,1)$ in spherical coordinates [see sketch in Fig. 1(a)]. The inverse mode volume of several eigenmodes with the angular momentum $l=7$ and transverse electric (TE) polarization, summed over the degenerate states with azimuthal number $m=-l, \ldots, l$, is shown in Fig. 1(a). The RSs of the sphere can be classified as leaky modes, whispering gallery modes (WGMs), and Fabry-Pérot (FP) modes, as indicated. The chosen dipole position is close to the field maximum of the fundamental WGM $(n=1)$, which as a result contributes with a small and nearly real mode volume. With increasing mode order and going into the FP modes, the mode volumes oscillate as the field maxima and minima move across the dipole position. Interestingly, the phase of the mode volumes rotates accordingly, yielding negative mode volumes at the positions of the field minima, at which the mode field is imaginary. This also illustrates that the radiative decay into the modes is not a simple superposition of Lorentzian lines describing independent channels, but instead shows interference. This is a consequence of the fact that modes of equal $l, m$, and polarization couple into the same outgoing loss channel.

The resulting partial PF for $l=7$ TE modes [see Fig. 1(b)] is dominated by the $n=1 \mathrm{WGM}$ providing on resonance a PF of about 20. The complex mode volume leads to nonLorentzian features in the spectrum, via the mode interference. The total contribution to the PF of all modes for each loss channel (for spherical symmetry all modes with equal $m, l$, and polarization) is strictly positive, as expected. To exemplify the issues with the LK normalization, we show the resulting PF for two finite integration volumes given by spheres of radius $R=$ $2 a$ and $R=30 a$. The observed deviation, which is increasing with $R$, is due to an underestimation of the contribution of leaky and FP modes and also leads to negative PF values, which are unphysical. Taking the limit $R \rightarrow \infty$, the mode volume diverges exponentially, according to Eq. (4), see Fig. 3 in Appendix C. This is also true for high- $Q$ modes but commences at larger $R$, so that the PF vanishes. In metallic resonators the modes have generally low $Q$ factors, yielding a fast exponential growth (see Fig. 4 in Appendix C) and significant errors of Eq. (4) for any $\mathcal{V}$.
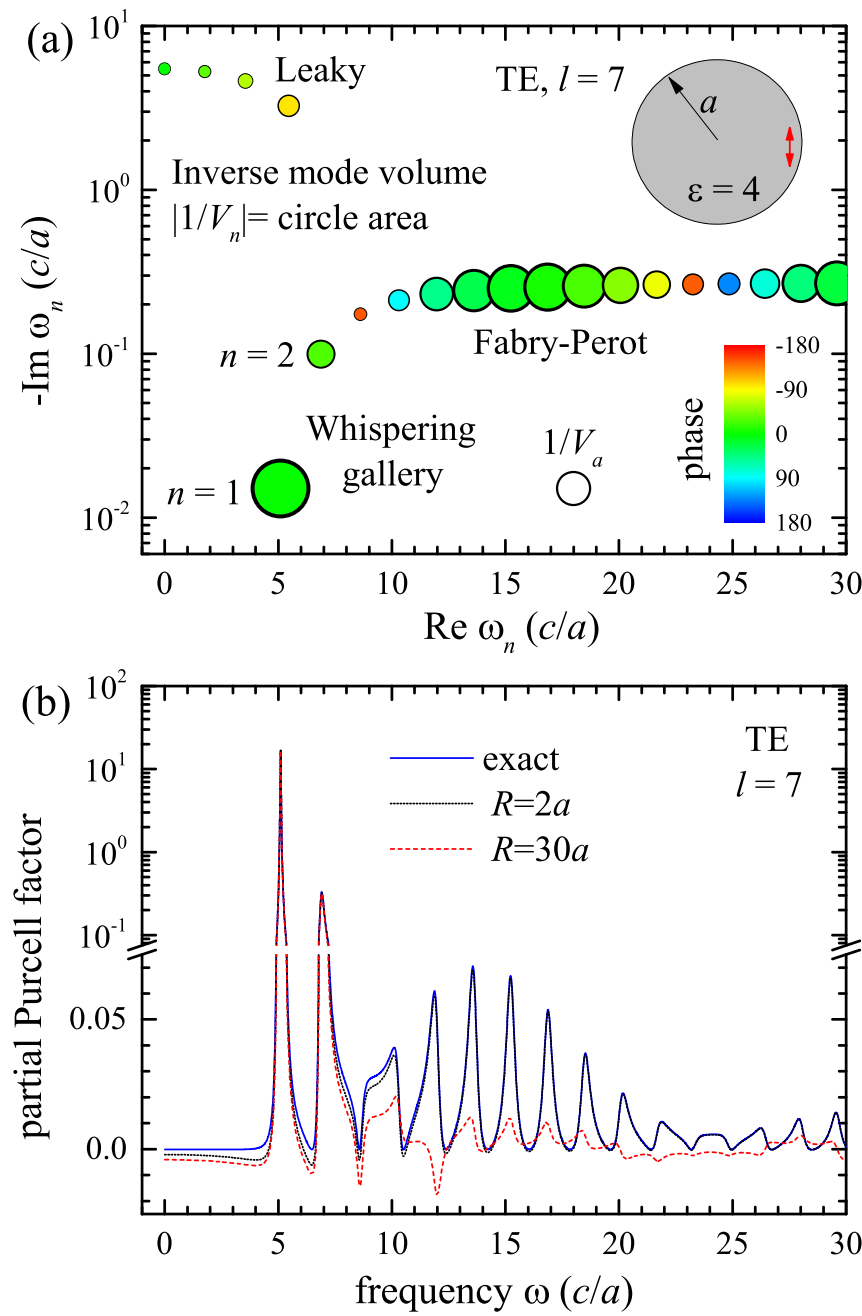

FIG. 1. (a) Mode volumes for a dielectric sphere in vacuum, with permittivity $\varepsilon=4$ and radius $a$, for $l=7 \mathrm{TE}$ modes and a point dipole placed at $\left|\mathbf{r}_{d}\right|=0.9 a$ with direction $\mathbf{e}=(0,0,1)$ in spherical coordinates (see sketch). The mode volume is presented as the sum of the inverse mode volume over all degenerate states $m=-l, \ldots, l$. Its amplitude is shown by the circle area and its phase by the color. The volume of the sphere $V_{a}=4 \pi a^{3} / 3$ is shown for comparison. The position of the circles in the complex frequency plane is given by the mode eigenfrequency $\omega_{n}$. (b) Partial Purcell factor calculated via Eq. (9) for $l=7$ and TE polarization, for the geometry of (a), using the exact mode normalization (blue line) and the LK normalization evaluated for integration volumes given by a sphere of radius $R=2 a$ and $R=30 a$, as labeled.

To exemplify the validity of the spectral representation based on the exact normalization, we compare the resulting PF with the one calculated using the analytic GF of a sphere. We take into account both TE and transverse magnetic (TM) polarizations and sum over all significant values of $l$ and $m$. Examples of mode volumes and partial PFs for the TM modes are shown in Figs. 5 and 6 of Appendix E for two different directions of the dipole. The resulting PF for a dipole at a distance $0.9 a$ from the center of the sphere averaged over its polarization directions is shown in Fig. 2, with partial PFs shown separately for TE modes in Figs. 2(a) and 2(b) and for TM modes in Fig. 2(b). In the low-frequency limit the well 

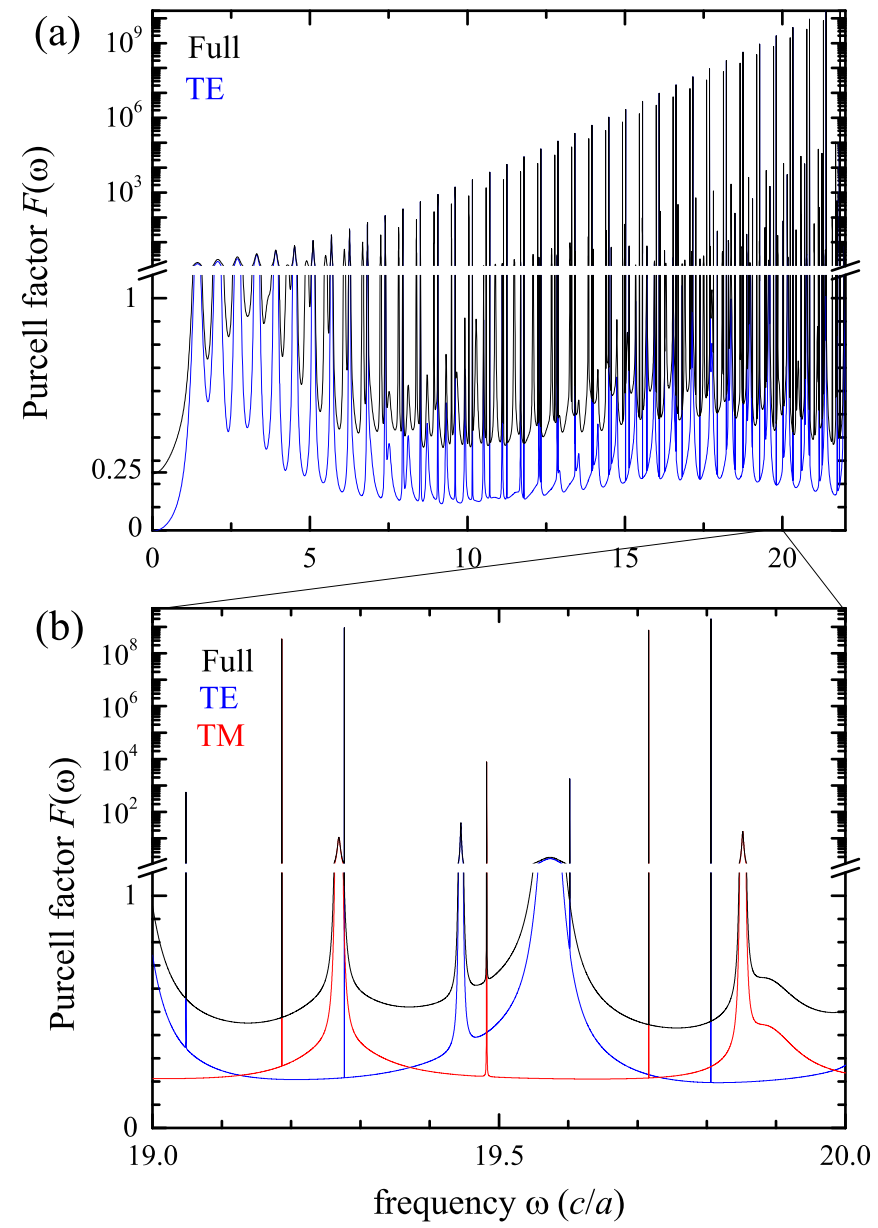

FIG. 2. Purcell factor versus transition frequency $\omega$ calculated by Eq. (9) using modes with $l<38$ and $\left|\omega_{n}\right|<40 c / a$. The dipole is placed at $\left|\mathbf{r}_{d}\right|=0.9 a$ as in Fig. 1, and the PF is averaged over the polarization directions. (a) Full PF (black line) and partial PF for TE modes only (blue line). (b) Zoom of (a), showing additionally the partial PF for TM modes (red line).

known static value of the field reduction by a factor of $3 /(2+\varepsilon)$ inside a dielectric sphere in vacuum is reproduced, leading to $F(0)=0.25$ for $\varepsilon=4$. Comparing these results with the ones obtained using the analytic GF of a sphere shows good agreement, as detailed in Appendix F. The spectral zoom in Fig. 2(b) allows one to see the extremely sharp WGM lines on top of much wider resonances and their separation into TE and TM modes. Purcell factors up to $10^{10}$ are found in resonance to WGM of similarly high $Q$ factors.

In conclusion, we have provided a general exact analytic form of the normalization of eigenmodes in an arbitrary finite open optical resonator. We have shown that this exact normalization can be used with high accuracy also for RSs determined by numerical solvers, while the flexible normalization volume it uses does not constrain the size of the computational domain at the same time. The correct normalization is of key importance for the electromagnetic theory, as it determines the spectral representation of the dyadic Green's function of Maxwell's wave equation, which can be used for calculation of any observable, such as scattering and extinction cross sections and local density of states [20].
This work was supported by the Cardiff University EPSRC Impact Acceleration Account EP/K503988/1, and the Sêr Cymru National Research Network in Advanced Engineering and Materials. The authors acknowledge discussions with T. Weiss and M. B. Doost, and thank G. Zorinyants for performing numerical calculations for the RSs of a nonspherical system using COMSOL.

\section{APPENDIX A: SPONTANEOUS EMISSION OF A QUANTUM DIPOLE IN AN ARBITRARY DIELECTRIC SYSTEM}

The full Hamiltonian describing a quantum dipole coupled to photon states in an arbitrary open optical system is given [15] by

$$
H=H_{0}+V \text {, }
$$

where

$$
H_{0}=\sum_{k} \hbar \omega_{k} a_{k}^{\dagger} a_{k}+\hbar \omega_{d} d^{\dagger} d
$$

is the noninteracting part and

$$
V=-i \sum_{k}\left(\varphi_{k} d^{\dagger} a_{k}-\varphi_{k}^{*} a_{k}^{\dagger} d\right)
$$

is the interaction between the dipole and photons in the rotating wave approximation. Here $a_{k}^{\dagger}$ is the bosonic photon creation operator in state $k, d^{\dagger}$ is the fermionic creation operator for the two-level system of the quantum dipole, with $\omega_{d}$ being the ground-to-excited state transition frequency, and

$$
\varphi_{k}=\sqrt{\frac{\hbar \omega_{k}}{2 \varepsilon_{0}}} \boldsymbol{\mu} \cdot \mathbf{f}_{k}\left(\mathbf{r}_{d}\right)
$$

is the coupling matrix element, in which $\boldsymbol{\mu}$ is the electric dipole moment of the point dipole placed at $\mathbf{r}=\mathbf{r}_{d}, \varepsilon_{0}$ is the vacuum permittivity, and $\mathbf{f}_{k}(\mathbf{r})$ is a vector eigenfunction of the electric field of the continuum state $k$ satisfying the Maxwell wave equation

$$
-\nabla \times \nabla \times \mathbf{f}_{k}(\mathbf{r})+\frac{\omega_{k}^{2}}{c^{2}} \hat{\boldsymbol{\epsilon}}(\mathbf{r}) \mathbf{f}_{k}(\mathbf{r})=0
$$

with a real eigenfrequency $\omega_{k} \geqslant 0$. The symmetric tensor $\hat{\boldsymbol{\epsilon}}(\mathbf{r})$ of the dielectric constant describes the open optical system under study and for simplicity is assumed here frequencyindependent.

Following Glauber [15], we consider the Schrödinger equation describing the full system ( $\hbar=1$ is used below for brevity of notations),

$$
i \frac{d}{d t}|\Phi(t)\rangle=H|\Phi(t)\rangle,
$$

and take its formal solution in the form

$$
|\Phi(t)\rangle=e^{-i H t}|\Phi(0)\rangle,
$$

where $|\Phi(t)\rangle$ is the wave function of the dipole-photon system. We are interested in the probability for the dipole to stay in the excited state and calculate the probability amplitude in the 
following way:

$$
\alpha(t)=\langle\Phi(0) \mid \Phi(t)\rangle=\left\langle 0\left|d e^{-i H t} d^{\dagger}\right| 0\right\rangle=\left\langle 0\left|d(t) U(t) d^{\dagger}\right| 0\right\rangle .
$$

In the above equation, the dipole moment operator is written in the interaction representation, $d(t)=e^{i H_{0} t} d e^{-i H_{0} t}$. We have also assumed that in the initial state, the photon subsystem is in its ground state and the quantum dipole is in its excited state, i.e., $|\Phi(0)\rangle=d^{\dagger}|0\rangle$, where $|0\rangle$ is the ground state of the full system. The evolution operator $U(t)=e^{i H_{0} t} e^{-i H t}$ satisfies the equation

$$
i \frac{d U(t)}{d t}=V(t) U(t)
$$

where $V(t)=e^{i H_{0} t} V e^{-i H_{0} t}$. Its solution can be written as an infinite perturbation series

$$
\begin{aligned}
U(t)= & 1+(-i) \int_{0}^{t} V\left(t_{1}\right) d t_{1} \\
& +(-i)^{2} \int_{0}^{t} V\left(t_{1}\right) d t_{1} \int_{0}^{t_{1}} V\left(t_{2}\right) d t_{2}+\cdots .
\end{aligned}
$$

To calculate $\alpha(t)$, we evaluate

$$
\begin{aligned}
& V\left(t_{1}\right) V\left(t_{2}\right) d^{\dagger}|0\rangle \\
&=-e^{i H_{0} t_{1}} \sum_{k_{1}}\left(\varphi_{k_{1}} d^{\dagger} a_{k_{1}}-\varphi_{k_{1}}^{*} a_{k_{1}}^{\dagger} d\right) \\
& \times e^{-i H_{0}\left(t_{1}-t_{2}\right)} \sum_{k_{2}}\left(\varphi_{k_{2}} d^{\dagger} a_{k_{2}}-\varphi_{k_{2}}^{*} a_{k_{2}}^{\dagger} d\right) e^{-i H_{0} t_{2}} d^{\dagger}|0\rangle \\
&= e^{i \omega_{d}\left(t_{1}-t_{2}\right)} \sum_{k}\left|\varphi_{k}\right|^{2} e^{-i \omega_{k}\left(t_{1}-t_{2}\right)} d^{\dagger}|0\rangle
\end{aligned}
$$

Then for $t>0, \alpha(t)$ can be written in the form of an integral equation:

$$
\begin{aligned}
\alpha(t)= & e^{-i \omega_{d} t}-\sum_{k}\left|\varphi_{k}\right|^{2} \int_{0}^{t} d t_{1} \int_{0}^{t_{1}} d t_{2} \\
& \times e^{-i \omega_{d}\left(t-t_{1}\right)} e^{-i \omega_{k}\left(t_{1}-t_{2}\right)} \alpha\left(t_{2}\right),
\end{aligned}
$$

which can be solved explicitly in the Fourier space,

$$
\tilde{\alpha}(\omega)=\frac{-i}{\omega-\omega_{d}-\Sigma(\omega)},
$$

where $\tilde{\alpha}(\omega)$ is the time Fourier transform of $\alpha(t)$, and the self-energy $\Sigma(\omega)$ is given by a formula

$$
\Sigma(\omega)=\frac{1}{\hbar^{2}} \sum_{k} \frac{\left|\varphi_{k}\right|^{2}}{\omega-\omega_{k}+i \delta_{+}},
$$

in which $\delta_{+} \rightarrow 0_{+}$and $\hbar$ has been restored. Note that the problem described by Eqs. (A1)-(A3) is the famous exactly solvable Fano-Anderson problem. Indeed, owing to the bilinear form of the interaction Eq. (A3) the exact perturbation series for the self-energy ends in first order [21].

Let us express the self-energy $\Sigma(\omega)$ in terms of the dyadic GF of Maxwell's wave equation. The full time-dependent GF $\hat{\mathcal{G}}\left(\mathbf{r}, \mathbf{r}^{\prime} ; t-t^{\prime}\right)$ satisfies the equation

$$
-\nabla \times \nabla \times \hat{\mathcal{G}}-\frac{\hat{\boldsymbol{\epsilon}}(\mathbf{r})}{c^{2}} \frac{\partial^{2} \hat{\mathcal{G}}}{\partial t^{2}}=\hat{\mathbf{1}} \delta\left(\mathbf{r}-\mathbf{r}^{\prime}\right) \delta\left(t-t^{\prime}\right)
$$

and has the following explicit form in terms of the continuum eigenstates, the solutions of Eq. (A5):

$$
\hat{\mathcal{G}}\left(\mathbf{r}, \mathbf{r}^{\prime} ; t-t^{\prime}\right)=\frac{c^{2}}{i} \sum_{k} \frac{\mathbf{f}_{k}(\mathbf{r}) \otimes \mathbf{f}_{k}^{*}\left(\mathbf{r}^{\prime}\right)}{2 \omega_{k}} e^{-i \omega_{k}\left|t-t^{\prime}\right|} .
$$

Note that substituting Eq. (A16) into Eq. (A15) and using Eq. (A5) results in the closure relation for the continuum eigenstates,

$$
\hat{\boldsymbol{\epsilon}}(\mathbf{r}) \sum_{k} \mathbf{f}_{k}(\mathbf{r}) \otimes \mathbf{f}_{k}^{*}\left(\mathbf{r}^{\prime}\right)=\hat{\mathbf{1}} \delta\left(\mathbf{r}-\mathbf{r}^{\prime}\right) .
$$

Fourier-transforming the GF given by Eq. (A16) versus $t-t^{\prime}$ we obtain

$$
\hat{\mathbf{G}}\left(\mathbf{r}, \mathbf{r}^{\prime} ; \omega\right)=c^{2} \sum_{k} \frac{\mathbf{f}_{k}(\mathbf{r}) \otimes \mathbf{f}_{k}^{*}\left(\mathbf{r}^{\prime}\right)}{\omega^{2}-\omega_{k}^{2}+i \delta_{+}} .
$$

Then, for a positive frequency $\omega$, we find, using Eq. (A4),

$$
\begin{aligned}
I\left(\mathbf{r}_{d}, \omega\right) & \equiv \boldsymbol{\mu} \cdot \operatorname{Im} \hat{\mathbf{G}}\left(\mathbf{r}_{d}, \mathbf{r}_{d} ; \omega\right) \boldsymbol{\mu} \\
& =-\frac{\pi \varepsilon_{0} c^{2}}{\hbar} \sum_{k} \frac{\left|\varphi_{k}\right|^{2}}{\omega_{k}^{2}} \delta\left(\omega-\omega_{k}\right),
\end{aligned}
$$

that allows us to express the self-energy in terms of the projection $I(\mathbf{r}, \omega)$ of the GF tensor:

$$
\begin{aligned}
\Sigma(\omega) & =\frac{1}{\hbar^{2}} \sum_{k} \int_{0}^{\infty} \frac{\left|\varphi_{k}\right|^{2} \delta\left(\omega^{\prime}-\omega_{k}\right) d \omega^{\prime}}{\omega-\omega^{\prime}+i \delta_{+}} \\
& =-\frac{1}{\pi \varepsilon_{0} c^{2} \hbar} \int_{0}^{\infty} \frac{I\left(\mathbf{r}_{d}, \omega^{\prime}\right) \omega^{\prime 2}}{\omega-\omega^{\prime}+i \delta_{+}} d \omega^{\prime} .
\end{aligned}
$$

For the GF of a homogeneous medium with the dielectric constant $\varepsilon$ we have

$$
\operatorname{Im} \hat{\mathbf{G}}^{0}(\mathbf{r}, \mathbf{r} ; \omega)=-\frac{\sqrt{\varepsilon} \omega}{6 \pi c} \hat{\mathbf{1}},
$$

as shown below, so that the integral in Eq. (A20) diverges for large $\omega^{\prime}$, which is the well known divergence problem of the Lamb shift, usually treated by introducing a frequency cutoff. For an inhomogeneous open optical system this integral is, however, convergent. Indeed, using the spectral representation of the GF in terms of resonant states (RSs) with complex eigenfrequencies $\omega_{n}[9,13]$

$$
\hat{\mathbf{G}}\left(\mathbf{r}, \mathbf{r}^{\prime} ; \omega\right)=c^{2} \sum_{n} \frac{\mathbf{E}_{n}(\mathbf{r}) \otimes \mathbf{E}_{n}\left(\mathbf{r}^{\prime}\right)}{2 \omega\left(\omega-\omega_{n}\right)}
$$

(see also Appendix B), we obtain for any $\mathbf{r}$ inside the system

$$
I(\mathbf{r}, \omega)=\frac{c^{2}}{2 \omega} \operatorname{Im} \sum_{n} \frac{g_{n}^{2}(\mathbf{r})}{\omega-\omega_{n}},
$$

where

$$
g_{n}(\mathbf{r})=\boldsymbol{\mu} \cdot \mathbf{E}_{n}(\mathbf{r}) .
$$

We note that RSs contribute to Eq. (A22) in pairs: Each RS $n$ with the eigenfrequency $\omega_{n}$ and electric field eigenfunction $\mathbf{E}_{n}(\mathbf{r})$ has a counterpart $-n$ with $\omega_{-n}=-\omega_{n}^{*}$ and $\mathbf{E}_{-n}(\mathbf{r})=$ 
$\mathbf{E}_{n}^{*}(\mathbf{r})$. Their joint contribution to Eq. (A23) is given by

$$
\begin{aligned}
\operatorname{Im} & {\left[\frac{g_{n}^{2}(\mathbf{r})}{\omega-\omega_{n}}+\frac{g_{n}^{* 2}(\mathbf{r})}{\omega+\omega_{n}^{*}}\right] } \\
& =\frac{A_{n}^{\prime \prime}\left(\omega-\omega_{n}^{\prime}\right)+A_{n}^{\prime} \omega_{n}^{\prime \prime}}{\left(\omega-\omega_{n}^{\prime}\right)^{2}+\omega_{n}^{\prime \prime 2}}+\frac{-A_{n}^{\prime \prime}\left(\omega+\omega_{n}^{\prime}\right)+A_{n}^{\prime} \omega_{n}^{\prime \prime}}{\left(\omega+\omega_{n}^{\prime}\right)^{2}+\omega_{n}^{\prime \prime}},
\end{aligned}
$$

(A25)

where $g_{n}^{2}(\mathbf{r})=A_{n}^{\prime}+i A_{n}^{\prime \prime}$ and $\omega_{n}=\omega_{n}^{\prime}+i \omega_{n}^{\prime \prime}$. Therefore $I(\mathbf{r}, \omega) \propto 1 / \omega^{3}$ at $\omega \rightarrow \infty$ and the integral in Eq. (A20) converges.

For small values of $\omega$, it is more practical to use a different form of the GF $[9,13]$

$$
\hat{\mathbf{G}}\left(\mathbf{r}, \mathbf{r}^{\prime} ; \omega\right)=c^{2} \sum_{n} \frac{\mathbf{E}_{n}(\mathbf{r}) \otimes \mathbf{E}_{n}\left(\mathbf{r}^{\prime}\right)}{2 \omega_{n}\left(\omega-\omega_{n}\right)},
$$

which follows from Eq. (A22) and the sum rule (see Appendix B)

$$
\sum_{n} \frac{\mathbf{E}_{n}(\mathbf{r}) \otimes \mathbf{E}_{n}\left(\mathbf{r}^{\prime}\right)}{\omega_{n}}=0 .
$$

Then $I(\mathbf{r}, \omega)$ can be written as

$$
I(\mathbf{r}, \omega)=\frac{c^{2}}{2} \operatorname{Im} \sum_{n} \frac{g_{n}^{2}(\mathbf{r})}{\omega_{n}\left(\omega-\omega_{n}\right)},
$$

and the contribution of the pair of poles takes the form

$$
\begin{aligned}
\operatorname{Im} & {\left[\frac{g_{n}^{2}(\mathbf{r})}{\omega_{n}\left(\omega-\omega_{n}\right)}-\frac{g_{n}^{* 2}(\mathbf{r})}{\omega_{n}^{*}\left(\omega+\omega_{n}^{*}\right)}\right] } \\
& =\frac{B_{n}^{\prime \prime}\left(\omega-\omega_{n}^{\prime}\right)+B_{n}^{\prime} \omega_{n}^{\prime \prime}}{\left(\omega-\omega_{n}^{\prime}\right)^{2}+\omega_{n}^{\prime \prime 2}}-\frac{-B_{n}^{\prime \prime}\left(\omega+\omega_{n}^{\prime}\right)+B_{n}^{\prime} \omega_{n}^{\prime \prime}}{\left(\omega+\omega_{n}^{\prime}\right)^{2}+\omega_{n}^{\prime \prime 2}},
\end{aligned}
$$

where $g_{n}^{2}(\mathbf{r}) / \omega_{n}=B_{n}^{\prime}+i B_{n}^{\prime \prime}$, so that $I(\mathbf{r}, \omega) \propto \omega$ in the limit $\omega \rightarrow 0$.

Note that for the poles of the GF on the imaginary $\omega$ axis which do not have counterparts, $g_{n}^{2}(\mathbf{r})$ is real, and both lowand high-frequency asymptotics of $I(\mathbf{r}, \omega)$ obtained above are preserved. Moreover, for the same reason, static modes (having $\omega_{n}=0$ ) do not contribute to the spontaneous emission, as the corresponding term of the GF is purely real (the modes are localized).

Using Eqs. (A23) or (A28), $\Sigma(\omega)$ can be calculated analytically for any finite number of RSs, thus providing direct access to the analytic continuation of $\tilde{\alpha}(\omega)$ into the complex $\omega$ plane and to its pole structure. Owing to the causality principle, $\tilde{\alpha}(\omega)$ has poles only in the lower half plane, which results in the following expression for the probability amplitude in the time domain:

$$
\alpha(t)=\theta(t) \sum_{j} c_{j} e^{-i \omega_{j} t},
$$

where

$$
\frac{1}{c_{j}}=1-\left.\frac{d \Sigma(\omega)}{d \omega}\right|_{\omega=\omega_{j}}
$$

and $\omega_{j}=\omega_{d}+\delta \omega_{j}-i \gamma_{j}$ are the poles of $\tilde{\alpha}(\omega)$. Such an analysis is important for the strong coupling regime. In the weak coupling regime instead, $\Sigma(\omega)$ can be considered as a small correction, and $\tilde{\alpha}(\omega)$ can be treated in the single-pole approximation leading to

$$
\alpha(t)=\theta(t) e^{-i\left(\omega_{d}+\delta \omega\right) t-\gamma t},
$$

where $\delta \omega-i \gamma=\Sigma\left(\omega_{d}\right)$ is the self-energy correction to the pole of GF of the dipole, calculated "on-shell," i.e., at $\omega=\omega_{d}$. The Lamb shift $\delta \omega$ and the spontaneous emission rate $\gamma$ then take the following explicit form:

$$
\begin{gathered}
\delta \omega=\frac{1}{\pi \varepsilon_{0} c^{2} \hbar} \int_{0}^{\infty} \frac{I\left(\mathbf{r}_{d}, \omega\right) \omega^{2} d \omega}{\omega-\omega_{d}}, \\
\gamma\left(\omega_{d}\right)=-\frac{\omega_{d}^{2}}{\varepsilon_{0} c^{2} \hbar} I\left(\mathbf{r}_{d}, \omega_{d}\right),
\end{gathered}
$$

where the principal value integral is introduced in Eq. (A33). Equations (A32)-(A34) are know in the literature as the Weisskopf-Wigner approximation [16].

Let us check that Eq. (A34) produces the correct expression for the spontaneous emission rate in the case of a homogeneous dielectric medium. The GF of the free space satisfies the equation

$-\nabla \times \nabla \times \hat{\mathbf{G}}^{0}\left(\mathbf{r}, \mathbf{r}^{\prime} ; \omega\right)+k^{2} \hat{\mathbf{G}}^{0}\left(\mathbf{r}, \mathbf{r}^{\prime} ; \omega\right)=\hat{\mathbf{1}} \delta\left(\mathbf{r}-\mathbf{r}^{\prime}\right)$,

where $k^{2}=\varepsilon \omega^{2} / c^{2}$ and $\varepsilon$ is the dielectric constant of the medium. The solution of Eq. (A35) has the form [19]

$$
\hat{\mathbf{G}}^{0}\left(\mathbf{r}, \mathbf{r}^{\prime} ; \omega\right)=-\left(\hat{\mathbf{1}}+\frac{1}{k^{2}} \nabla \otimes \nabla\right) \frac{e^{i k\left|\mathbf{r}-\mathbf{r}^{\prime}\right|}}{4 \pi\left|\mathbf{r}-\mathbf{r}^{\prime}\right|},
$$

or, more explicitly [22],

$$
\hat{\mathbf{G}}^{0}\left(\mathbf{r}, \mathbf{r}^{\prime} ; \omega\right)=C \hat{\mathbf{1}}+D \frac{\mathbf{b} \otimes \mathbf{b}}{b^{2}},
$$

where $\mathbf{b}=\mathbf{r}-\mathbf{r}^{\prime}, b=|\mathbf{b}|$, and

$$
\begin{gathered}
C=-\left(1+\frac{i k b-1}{k^{2} b^{2}}\right) \frac{e^{i k b}}{4 \pi b}=\frac{2-k^{2} b^{2}}{8 \pi k^{2} b^{3}}-i \frac{k}{6 \pi}+k O(k b), \\
D=-\frac{3-3 i k b-k^{2} b^{2}}{k^{2} b^{2}} \frac{e^{i k b}}{4 \pi b}=-\frac{6+k^{2} b^{2}}{8 \pi k^{2} b^{3}}+k O(k b),
\end{gathered}
$$

expanded up to zeroth order in $k b$. Taking the limit $\mathbf{r}^{\prime} \rightarrow \mathbf{r}$, so that $b \rightarrow 0$, we obtain Eq. (A21) and finally

$$
\gamma_{0}(\omega)=-\frac{\omega^{2}}{\varepsilon_{0} \hbar c^{2}} \boldsymbol{\mu} \cdot \operatorname{Im} \hat{\mathbf{G}}^{0}\left(\mathbf{r}_{d}, \mathbf{r}_{d} ; \omega\right) \boldsymbol{\mu}=\frac{\sqrt{\varepsilon} \omega^{3} \mu^{2}}{6 \pi \epsilon_{0} \hbar c^{3}},
$$

in agreement with [1]. Note that using the spectral representation of the GF in the form of Eq. (A26), the spontaneous decay rate $\gamma(\omega)$ of an inhomogeneous open optical system also scales like $\omega^{3}$ at $\omega \rightarrow 0\left[I\left(\mathbf{r}_{d}, \omega\right) \propto \omega\right.$ as shown above $]$. This makes the PF $F(\omega)=\gamma(\omega) / \gamma_{0}(\omega)$ finite at $\omega \rightarrow 0$. 


\section{APPENDIX B: SPECTRAL REPRESENTATION OF THE GREEN'S FUNCTION AND NORMALIZATION OF RESONANT STATES}

The Green's function $\hat{\mathbf{G}}\left(\mathbf{r}, \mathbf{r}^{\prime} ; \omega\right)$ of an open optical system is a tensor which satisfies Maxwell's wave equation with a delta-function source term (below $c=1$ is used for brevity of notations),

$$
-\nabla \times \nabla \times \hat{\mathbf{G}}\left(\mathbf{r}, \mathbf{r}^{\prime} ; \omega\right)+\omega^{2} \hat{\boldsymbol{\epsilon}}(\mathbf{r} ; \omega) \hat{\mathbf{G}}\left(\mathbf{r}, \mathbf{r}^{\prime} ; \omega\right)=\hat{\mathbf{1}} \delta\left(\mathbf{r}-\mathbf{r}^{\prime}\right),
$$

and outgoing wave boundary conditions. Treating $\hat{\mathbf{G}}\left(\mathbf{r}, \mathbf{r}^{\prime} ; \omega\right)$ as a function of a complex $\omega$ we use the fact that the GF has a countable number of simple poles in the lower half plane at $\omega=\tilde{\omega}_{n}$. We further note that for $\omega \rightarrow \infty$, the GF vanishes inside the area of inhomogeneity of $\hat{\boldsymbol{\epsilon}}(\mathbf{r} ; \omega)$. Note that the frequency dependence of the permittivity tensor is included. Then according to the Mittag-Leffler theorem [23,24] we can write

$$
\hat{\mathbf{G}}\left(\mathbf{r}, \mathbf{r}^{\prime} ; \omega\right)=\sum_{n} \frac{\hat{\mathbf{R}}_{n}\left(\mathbf{r}, \mathbf{r}^{\prime}\right)}{\omega-\tilde{\omega}_{n}}
$$

where $\hat{\mathbf{R}}_{n}\left(\mathbf{r}, \mathbf{r}^{\prime}\right)$ is the residue of the GF at $\omega=\tilde{\omega}_{n}$.

Now, for each RS having the eigenfrequency $\omega_{n}$ and the electric field $\mathbf{E}_{n}(\mathbf{r})$ satisfying the homogeneous Maxwell wave equation

$$
-\nabla \times \nabla \times \mathbf{E}_{n}(\mathbf{r})+\omega_{n}^{2} \hat{\boldsymbol{\epsilon}}\left(\mathbf{r} ; \omega_{n}\right) \mathbf{E}_{n}(\mathbf{r})=0
$$

and outgoing or incoming wave boundary conditions, we introduce an analytic continuation $\mathbf{F}_{n}(\mathbf{r}, \omega)$, such that

$$
\lim _{\omega \rightarrow \omega_{n}} \mathbf{F}_{n}(\mathbf{r}, \omega)=\mathbf{E}_{n}(\mathbf{r})
$$

$\mathbf{F}_{n}(\mathbf{r}, \omega)$ is defined as a solution of the inhomogeneous Maxwell wave equations

$$
-\nabla \times \nabla \times \mathbf{F}_{n}(\mathbf{r} ; \omega)+\omega^{2} \hat{\boldsymbol{\epsilon}}(\mathbf{r} ; \omega) \mathbf{F}_{n}(\mathbf{r} ; \omega)=\left(\omega^{2}-\omega_{n}^{2}\right) \boldsymbol{\sigma}_{n}(\mathbf{r}),
$$

in which $\sigma_{n}(\mathbf{r})$ is an arbitrary function vanishing outside the system and normalized in such a way that

$$
\int_{\mathcal{V}} \mathbf{E}_{n}(\mathbf{r}) \cdot \boldsymbol{\sigma}_{n}(\mathbf{r}) d \mathbf{r}=1,
$$

where $\mathcal{V}$ is an arbitrary simply connected volume including all the inhomogeneities of $\hat{\boldsymbol{\epsilon}}(\mathbf{r} ; \omega)$. In the case of degenerate modes, $\omega_{m}=\omega_{n}$ for $m \neq n$, the source $\sigma_{n}(\mathbf{r})$ has to be chosen in such a way that, additionally, $\int_{\mathcal{V}} \mathbf{E}_{m}(\mathbf{r}) \cdot \boldsymbol{\sigma}_{n}(\mathbf{r}) d \mathbf{r}=0$. Solving Eq. (B5) with the help of the GF Eq. (B2) we obtain

$$
\mathbf{F}_{n}(\mathbf{r} ; \omega)=\sum_{n^{\prime}} \frac{\omega^{2}-\omega_{n}^{2}}{\omega-\tilde{\omega}_{n^{\prime}}} \int_{\mathcal{V}} \hat{\mathbf{R}}_{n^{\prime}}\left(\mathbf{r}, \mathbf{r}^{\prime}\right) \boldsymbol{\sigma}_{n}\left(\mathbf{r}^{\prime}\right) d \mathbf{r}^{\prime} .
$$

Taking the limit of Eq. (B4) and using the fact that $\hat{\mathbf{G}}\left(\mathbf{r}, \mathbf{r}^{\prime} ; \omega\right)$ is a symmetric tensor, which follows from the reciprocity theorem [25], we find

$$
\hat{\mathbf{R}}_{n}\left(\mathbf{r}, \mathbf{r}^{\prime}\right)=\frac{\mathbf{E}_{n}(\mathbf{r}) \otimes \mathbf{E}_{n}\left(\mathbf{r}^{\prime}\right)}{2 \omega_{n}}
$$

and $\omega_{n}=\tilde{\omega}_{n}$, leading to the spectral representation Eq. (A26). Substituting it into Eq. (B1) and using Eq. (B3) results in the closure relation

$$
\sum_{n} \frac{\omega^{2} \hat{\boldsymbol{\epsilon}}(\mathbf{r} ; \omega)-\omega_{n}^{2} \hat{\boldsymbol{\epsilon}}\left(\mathbf{r} ; \omega_{n}\right)}{2 \omega_{n}\left(\omega-\omega_{n}\right)} \mathbf{E}_{n}(\mathbf{r}) \otimes \mathbf{E}_{n}\left(\mathbf{r}^{\prime}\right)=\hat{\mathbf{1}} \delta\left(\mathbf{r}-\mathbf{r}^{\prime}\right),
$$

which splits into sum rules, including Eq. (A27), and a simpler closure relation [14]

$$
\frac{1}{2} \sum_{n} \hat{\boldsymbol{\epsilon}}\left(\mathbf{r} ; \omega_{n}\right) \mathbf{E}_{n}(\mathbf{r}) \otimes \mathbf{E}_{n}\left(\mathbf{r}^{\prime}\right)=\hat{\mathbf{1}} \delta\left(\mathbf{r}-\mathbf{r}^{\prime}\right) .
$$

As already noted in Appendix A, combining Eq. (A26) and the sum rule Eq. (A27) leads to an alternative form of the spectral representation Eq. (A22) which was used in the RSE $[9,13]$.

The form of the GF Eq. (A26) determines the normalization of RSs which technically follows from Eq. (B6) by substituting $\sigma_{n}(\mathbf{r})$ from Eq. (B5) and taking the limit $\omega \rightarrow \omega_{n}$ (below the $\operatorname{argument} \mathbf{r}$ is omitted for brevity):

$$
\begin{aligned}
1= & \int_{\mathcal{V}} d \mathbf{r} \mathbf{E}_{n} \cdot \boldsymbol{\sigma}_{n} \\
= & \lim _{\omega \rightarrow \omega_{n}} \int_{\mathcal{V}} d \mathbf{r} \mathbf{E}_{n} \cdot \frac{-\nabla \times \nabla \times \mathbf{F}_{n}+\omega^{2} \hat{\boldsymbol{\epsilon}}(\omega) \mathbf{F}_{n}}{\omega^{2}-\omega_{n}^{2}} \\
& -\lim _{\omega \rightarrow \omega_{n}} \int_{\mathcal{V}} d \mathbf{r} \mathbf{F}_{n} \cdot \frac{-\nabla \times \nabla \times \mathbf{E}_{n}+\omega_{n}^{2} \hat{\boldsymbol{\epsilon}}\left(\omega_{n}\right) \mathbf{E}_{n}}{\omega^{2}-\omega_{n}^{2}} \\
= & \lim _{\omega \rightarrow \omega_{n}} \int_{\mathcal{V}} d \mathbf{r} \mathbf{F}_{n} \cdot \frac{\omega^{2} \hat{\boldsymbol{\epsilon}}(\omega)-\omega_{n}^{2} \hat{\boldsymbol{\epsilon}}\left(\omega_{n}\right)}{\omega^{2}-\omega_{n}^{2}} \mathbf{E}_{n} \\
& +\lim _{\omega \rightarrow \omega_{n}} \frac{\int_{\mathcal{V}}\left(\mathbf{F}_{n} \cdot \nabla \times \nabla \times \mathbf{E}_{n}-\mathbf{E}_{n} \cdot \nabla \times \nabla \times \mathbf{F}_{n}\right) d \mathbf{r}}{\omega^{2}-\omega_{n}^{2}} \\
= & \left.\int_{\mathcal{V}} d \mathbf{r} \mathbf{E}_{n} \cdot \frac{\partial\left(\omega^{2} \hat{\boldsymbol{\epsilon}}(\omega)\right)}{\partial\left(\omega^{2}\right)}\right|_{\omega=\omega_{n}} \mathbf{E}_{n} \\
& +\lim _{\omega \rightarrow \omega_{n}} \frac{\oint_{S_{\mathcal{V}}} d S\left(\mathbf{E}_{n} \cdot \frac{\partial \mathbf{F}_{n}}{\partial s}-\mathbf{F}_{n} \cdot \frac{\partial \mathbf{E}_{n}}{\partial s}\right)}{\omega^{2}-\omega_{n}^{2}},
\end{aligned}
$$

where after using some vector algebra we have applied the divergence theorem to convert a volume integral into a surface integral over the closed surface $S_{\mathcal{V}}$, the boundary of $\mathcal{V}$, with $\partial / \partial s$ denoting the directional derivative normal to this surface. We have also used the fact that $\nabla \cdot \mathbf{E}_{n}=\nabla \cdot \mathbf{F}_{n}=0$ outside the system.

For any surface $S_{\mathcal{V}}$, the limit in the last term in Eq. (B11) can be evaluated explicitly by using the functional dependence of the electric field outside the system, where $\hat{\boldsymbol{\epsilon}}(\mathbf{r})=\hat{\mathbf{1}}$ up to a scalar constant. For any mode with $\omega_{n} \neq 0$, the RS wave function outside the system is given by $\mathbf{E}_{n}(\mathbf{r})=\mathbf{Q}_{n}\left(\omega_{n} \mathbf{r}\right)$, where $\mathbf{Q}_{n}(\mathbf{q})$ is a vector function satisfying the equation

$$
\nabla_{\mathbf{q}} \times \nabla_{\mathbf{q}} \times \mathbf{Q}_{n}(\mathbf{q})=\mathbf{Q}_{n}(\mathbf{q})
$$

and the proper boundary conditions at system interfaces and at $\mathbf{q} \rightarrow \infty$. The analytic continuation of $\mathbf{E}_{n}(\mathbf{r})$ can therefore be taken in the form

$$
\mathbf{F}_{n}(\mathbf{r}, \omega)=\mathbf{Q}_{n}(\omega \mathbf{r}) .
$$

In doing this, one could require, for example, that $\mathbf{F}_{n}(\mathbf{r}, \omega)$ outside the system has the same expansion in terms of vector spherical harmonics as $\mathbf{E}_{n}(\mathbf{r})$ itself, so that the frequency 
dependence of $\mathbf{F}_{n}(\mathbf{r}, \omega)$ comes only from the argument $\omega \mathbf{r}$ of the vector spherical harmonics, and not from the expansion coefficients. This imposes certain condition on the choice of $\sigma_{n}(\mathbf{r})$. It can be shown, however, that using instead an arbitrary frequency dependence of the coefficients in the expansion of $\mathbf{F}_{n}(\mathbf{r}, \omega)$ does not change the value of the limit in Eq. (B11), so that $\sigma_{n}(\mathbf{r})$ can again be an arbitrary function vanishing outside the system.

Now, using the Taylor expansion of Eq. (B13) about the point $\omega=\omega_{n}$,

$$
\begin{aligned}
\mathbf{F}_{n}(\mathbf{r}, \omega) & \approx \mathbf{Q}_{n}\left(\omega_{n} \mathbf{r}\right)+\left.\left(\omega-\omega_{n}\right) \frac{\partial \mathbf{Q}_{n}(\omega \mathbf{r})}{\partial \omega}\right|_{\omega=\omega_{n}} \\
& =\mathbf{E}_{n}(\mathbf{r})+\frac{\omega-\omega_{n}}{\omega_{n}}(\mathbf{r} \cdot \nabla) \mathbf{E}_{n}(\mathbf{r}),
\end{aligned}
$$

and substituting it into Eq. (B11), we obtain

$$
\begin{aligned}
1= & \left.\int_{\mathcal{V}} d \mathbf{r} \mathbf{E}_{n} \cdot \frac{\partial\left(\omega^{2} \hat{\boldsymbol{\epsilon}}(\omega)\right)}{\partial\left(\omega^{2}\right)}\right|_{\omega=\omega_{n}} \mathbf{E}_{n} \\
& +\frac{c^{2}}{2 \omega_{n}^{2}} \oint_{S_{\mathcal{V}}} d S\left[\mathbf{E}_{n} \cdot \frac{\partial \mathbf{K}_{n}}{\partial s}-\mathbf{K}_{n} \cdot \frac{\partial \mathbf{E}_{n}}{\partial s}\right],
\end{aligned}
$$

where

$$
\mathbf{K}_{n}(\mathbf{r})=(\mathbf{r} \cdot \nabla) \mathbf{E}_{n}(\mathbf{r}),
$$

and the speed of light is restored.

We note that the normalization of static modes $\left(\omega_{n}=0\right)$ is different and has been treated in [13]. They do not contribute to the radiative decay, as explained in Appendix A, and thus are not further considered here.

In the absence of dispersion, the first integral in the normalization Eq. (B15) is simplified to $\int_{\mathcal{V}} d \mathbf{r} \mathbf{E}_{n} \cdot \hat{\boldsymbol{\epsilon}} \mathbf{E}_{n}$, yielding Eq. (8) of the main text. Using also spherical coordinates in the surface integral, Eq. (B15) reduces to

$$
\begin{aligned}
1= & \int_{\mathcal{V}} d \mathbf{r} \mathbf{E}_{n} \cdot \hat{\boldsymbol{\epsilon}} \mathbf{E}_{n} \\
& +\frac{c^{2}}{2 \omega_{n}^{2}} \oint_{S_{\mathcal{V}}} d S\left[\mathbf{E}_{n} \cdot \frac{\partial}{\partial s} r \frac{\partial \mathbf{E}_{n}}{\partial r}-r \frac{\partial \mathbf{E}_{n}}{\partial r} \cdot \frac{\partial \mathbf{E}_{n}}{\partial s}\right],
\end{aligned}
$$

where $r=|\mathbf{r}|$, the radius in spherical coordinates. Finally, if $\hat{\boldsymbol{\epsilon}}(\mathbf{r})=\hat{\mathbf{1}} \varepsilon(\mathbf{r})$ and the surface of integration is chosen in the form of a sphere of radius $R$ with the center at the origin, the normalization Eq. (B15) takes its original form published in [9]:

$$
\begin{aligned}
1= & \int_{\mathcal{V}_{R}} \varepsilon(\mathbf{r}) \mathbf{E}_{n}^{2} d \mathbf{r} \\
& +\frac{c^{2}}{2 \omega_{n}^{2}} \oint_{S_{R}}\left[\mathbf{E}_{n} \cdot \frac{\partial}{\partial r} r \frac{\partial \mathbf{E}_{n}}{\partial r}-r\left(\frac{\partial \mathbf{E}_{n}}{\partial r}\right)^{2}\right] d S,
\end{aligned}
$$

where $\mathcal{V}_{R}$ is the volume and $S_{R}$ is the surface area of the sphere. Let us also note that if the homogeneous space outside the system is not vacuum but a medium described by a frequencydependent uniform permittivity $\varepsilon(\omega)$, the surface term of the normalization Eq. (B15) acquires an additional factor

$$
\left.\frac{1}{\varepsilon(\omega)} \frac{\partial\left(\omega^{2} \varepsilon(\omega)\right)}{\partial\left(\omega^{2}\right)}\right|_{\omega=\omega_{n}}
$$

which can be easily obtained following the derivation in Eq. (B14). Obviously this factor is equal to 1 for nondispersive media.

The normalization of RSs with the surface term as given, e.g., in Eq. (B15) contains second-order spatial derivatives of the RS field. In some numerical implementations like those considered in Appendix G below, using second-order derivatives can lead to an accumulation of numerical errors. It is therefore useful to provide a version of normalization which contains only first derivatives of the field. This can be obtained by noting that the surface integral in Eq. (B15) actually presents the flux through a closed surface $S_{\mathcal{V}}$ of the field

$$
\Phi_{1}(\mathbf{r})=\sum_{i=x, y, z}\left(E_{i} \nabla K_{i}-K_{i} \nabla E_{i}\right)
$$

in which $\mathbf{E}=\mathbf{E}_{n}$ and $\mathbf{K}=\mathbf{K}_{n}$ defined by Eq. (B16). Then, using Gauss's theorem, this surface integral can then be transformed to the flux of another field $\Phi_{2}$ linked to the first one through

$$
\Phi_{1}=\Phi_{2}+\nabla \times \mathbf{A},
$$

where $\mathbf{A}(\mathbf{r})$ is an arbitrary field. By representing $\Phi_{1}$ as

$$
\Phi_{1}=\nabla(\mathbf{E} \cdot \mathbf{K})-2 \sum_{i=x, y, z} K_{i} \nabla E_{i},
$$

and then using the fact that

$$
\begin{aligned}
2 \nabla(\mathbf{E} \cdot \mathbf{K}) & =\nabla(\mathbf{r} \cdot \nabla) E^{2} \\
& =-\nabla E^{2}+\mathbf{r} \nabla^{2} E^{2}-\nabla \times\left(\mathbf{r} \times \nabla E^{2}\right),
\end{aligned}
$$

where $E^{2}=\mathbf{E} \cdot \mathbf{E}$, the second-order derivatives of the E field are partly removed from Eq. (B22) by the curl. The remaining term containing $\nabla^{2} E^{2}$ can be transformed to lower orders by applying Maxwell's wave equation in vacuum, $\nabla^{2} \mathbf{E}=-k^{2} \mathbf{E}$, so that

$$
\frac{1}{2} \nabla^{2} E^{2}=-k^{2} E^{2}+\sum_{i, j=x, y, z}\left(\frac{\partial E_{i}}{\partial x_{j}}\right)^{2} .
$$

Then we obtain

$$
\mathbf{A}(\mathbf{r})=-\frac{1}{2} \mathbf{r} \times \nabla E^{2}
$$

and

$$
\Phi_{2}=-\frac{1}{2} \nabla E^{2}-k^{2} \mathbf{r} E^{2}+\mathbf{r} \sum_{i j}\left(\frac{\partial E_{i}}{\partial x_{j}}\right)^{2}-2 \sum_{i} K_{i} \nabla E_{i}
$$

$(i, j=x, y, z)$, the latter not containing derivatives of the field $\mathbf{E}$ higher than first order. Omitting for simplicity of notation the dispersion in the volume integral, the modified normalization, equivalent to Eq. (B15) but containing only first derivatives of $\mathbf{E}_{n}(\mathbf{r})$ in the surface term, takes the form

$$
1=\int_{\mathcal{V}} d \mathbf{E}_{n} \cdot \hat{\boldsymbol{\epsilon}} \mathbf{E}_{n} d \mathbf{r}+\frac{c^{2}}{2 \omega_{n}^{2}} \oint_{S_{\mathcal{V}}} \Phi_{2} \cdot d \mathbf{S},
$$

where $\Phi_{2}(\mathbf{r})$ is given by Eq. (B26), with $\mathbf{E}(\mathbf{r})=\mathbf{E}_{n}(\mathbf{r})$ and $\mathbf{K}(\mathbf{r})=\mathbf{K}_{n}(\mathbf{r})$ defined by Eq. (B16).

The orthogonality of two RSs with indices $n$ and $m$ has the form similar to the normalization Eq. (B11) or Eq. (B15) 
and can be obtained by multiplying Eq. (B3) with $\mathbf{E}_{m}(\mathbf{r})$, integrating over the volume $\mathcal{V}$, and subtracting a similar result with $n$ and $m$ swapped. This leads, after a similar transformation to the surface term made with help of the divergence theorem, to

$$
\begin{aligned}
0= & \int_{\mathcal{V}} d \mathbf{r} \mathbf{E}_{n} \cdot\left[\omega_{m}^{2} \hat{\boldsymbol{\epsilon}}\left(\omega_{m}\right)-\omega_{n}^{2} \hat{\boldsymbol{\epsilon}}\left(\omega_{n}\right)\right] \mathbf{E}_{m} \\
& +\oint_{S_{\mathcal{V}}} d S\left[\mathbf{E}_{n} \cdot \frac{\partial \mathbf{E}_{m}}{\partial s}-\mathbf{E}_{m} \cdot \frac{\partial \mathbf{E}_{n}}{\partial s}\right] .
\end{aligned}
$$

\section{APPENDIX C: LEUNG-KRISTENSEN NORMALIZATION}

Following Leung et al. [7], Kristensen et al. [4] have introduced a normalization of RSs in the form of Eq. (4) of the main text, which we call here LK normalization. We found that this normalization is only correct for so-called $s$ waves, i.e., $l=0$ modes of a spherically symmetric system, where $l$ is the orbital quantum number. However, owing to the vectorial nature of the electromagnetic field, $l=0$ eigenmodes do not exist, so that the LK normalization is incorrect for all modes in electrodynamics.

We illustrate this finding for TE modes of a dielectric sphere. We compare the mode volume $V_{n}^{\mathrm{LK}}$, calculated using the LK normalization for a finite spherical domain $\mathcal{V}_{R}$, with the correct one, $V_{n}$, calculated using the exact normalization Eq. (8). To do so, we introduce the ratio $N_{n}(R)$ between the two mode volumes so that

$$
V_{n}^{\mathrm{LK}}=V_{n} N_{n}(R) \text {. }
$$

This ratio is given by the sum of the volume and surface normalization integrals in Eq. (4):

$$
N_{n}(R)=I_{n}(R)+S_{n}(R)
$$

with

$$
I_{n}(R)=\int_{\mathcal{V}_{R}} \varepsilon(\mathbf{r}) \mathbf{E}_{n}^{2}(\mathbf{r}) d \mathbf{r}
$$

and

$$
S_{n}(R)=\frac{i}{2 k_{n}} \oint_{S_{R}} \mathbf{E}_{n}^{2}(\mathbf{r}) d S .
$$

Here $\mathcal{V}_{R}$ is the volume of a sphere of radius $R, S_{R}$ is its surface, and $k_{n}=\omega_{n} / c$ is the RS wave number. For the LK normalization to be correct, the factor $N_{n}(R)$ has to converge to unity in the limit $R \rightarrow \infty$. To show that this is not the case we derive the resulting error at finite $R$ by calculating $N_{n}(R)$ for the correctly normalized $\mathbf{E}_{n}(\mathbf{r})$ and consider the limit $R \rightarrow \infty$.

For a dielectric sphere of radius $a$ in vacuum, described by the dielectric constant

$$
\varepsilon(r)=\left\{\begin{array}{cc}
n_{r}^{2} & \text { for } r \leqslant a, \\
1 & \text { for } r>a,
\end{array}\right.
$$

the eigenfunctions of the TE modes normalized via Eq. (8) have the form (in spherical polar coordinates) [13]

$$
\mathbf{E}_{n}^{\mathrm{TE}}(\mathbf{r})=A_{l} R_{l}\left(r, k_{n}\right)\left(\begin{array}{c}
0 \\
\frac{1}{\sin \theta} \frac{\partial}{\partial \varphi} Y_{l m}(\Omega) \\
-\frac{\partial}{\partial \theta} Y_{l m}(\Omega)
\end{array}\right),
$$

where $Y_{l m}(\Omega)$ are the spherical harmonics,

$$
R_{l}(r, k)=\left\{\begin{array}{lll}
j_{l}\left(n_{r} k r\right) / j_{l}\left(n_{r} k a\right) & \text { for } \quad r \leqslant a, \\
h_{l}(k r) / h_{l}(k a) & \text { for } \quad r>a,
\end{array}\right.
$$

$j_{l}(z)$ and $h_{l}(z) \equiv h_{l}^{(1)}(z)$ are, respectively, the spherical Bessel and Hankel functions of first kind,

$$
A_{l}=\sqrt{\frac{2}{l(l+1) a^{3}\left(n_{r}^{2}-1\right)}}
$$

are normalization constants, and $k_{n}$ are the solutions of the secular equation

$$
\frac{n_{r} j_{l+1}\left(n_{r} k_{n} a\right)}{j_{l}\left(n_{r} k_{n} a\right)}=\frac{h_{l+1}\left(k_{n} a\right)}{h_{l}\left(k_{n} a\right)} .
$$

Using these properties, we evaluate the volume and surface normalization integrals for $R \geqslant a$ as

$$
\begin{aligned}
I_{n}(R)= & l(l+1) A_{l}^{2} \int_{0}^{R} R_{l}^{2}\left(r, k_{n}\right) \varepsilon(r) r^{2} d r \\
= & \frac{2}{a^{3}\left(n_{r}^{2}-1\right)}\left[n_{r}^{2} \int_{0}^{a} \frac{j_{l}^{2}\left(n_{r} k_{n} r\right)}{j_{l}^{2}\left(n_{r} k_{n} a\right)} r^{2} d r\right. \\
& \left.+\int_{a}^{R} \frac{h_{l}^{2}\left(k_{n} r\right)}{h_{l}^{2}\left(k_{n} a\right)} r^{2} d r\right] \\
= & 1+\frac{(R / a)^{3}}{n_{r}^{2}-1} \frac{h_{l}^{2}\left(k_{n} R\right)}{h_{l}^{2}\left(k_{n} a\right)}\left[1-\frac{h_{l-1}\left(k_{n} R\right) h_{l+1}\left(k_{n} R\right)}{h_{l}^{2}\left(k_{n} R\right)}\right]
\end{aligned}
$$

and

$$
S_{n}(R)=\frac{i R^{2}}{2 k_{n}} l(l+1) A_{l}^{2} R_{l}^{2}\left(R, k_{n}\right)=\frac{i}{k_{n} R} \frac{(R / a)^{3}}{n_{r}^{2}-1} \frac{h_{l}^{2}\left(k_{n} R\right)}{h_{l}^{2}\left(k_{n} a\right)},
$$

and consequently find

$$
N_{n}(R)=1+\frac{1}{n_{r}^{2}-1}\left(\frac{R}{a}\right)^{3} \frac{h_{l}^{2}\left(k_{n} R\right)}{h_{l}^{2}\left(k_{n} a\right)} Q_{n}(R),
$$

where

$$
Q_{n}(R)=1-\frac{h_{l-1}\left(k_{n} R\right) h_{l+1}\left(k_{n} R\right)}{h_{l}^{2}\left(k_{n} R\right)}+\frac{i}{k_{n} R} .
$$

To investigate the behavior of $N_{n}(R)$ for large $k_{n} R$, we use the asymptotic formula for $h_{l}(z)$ at large arguments:

$$
h_{l}(z)=(-i)^{l+1} \frac{e^{i z}}{z} \sum_{p=0}^{l} \frac{(l+p) !}{(l-p) ! p !} \frac{1}{(-2 i z)^{p}} .
$$

We find that in $Q_{n}(R)$, the 0th-, 1st-, and 2nd-order terms in $1 /\left(k_{n} R\right)$ are vanishing, so that

$$
Q_{n}(R)=\frac{C_{l}\left(k_{n} R\right)}{\left(k_{n} R\right)^{3}}
$$

and consequently

$$
\frac{V_{n}^{\mathrm{LK}}}{V_{n}}=N_{n}(R)=1+\frac{C_{l}\left(k_{n} R\right)}{\left(n_{r}^{2}-1\right)\left(k_{n} a\right)^{3} h_{l}^{2}\left(k_{n} a\right)} \frac{e^{2 i k_{n} R}}{\left(k_{n} R\right)^{2}},
$$



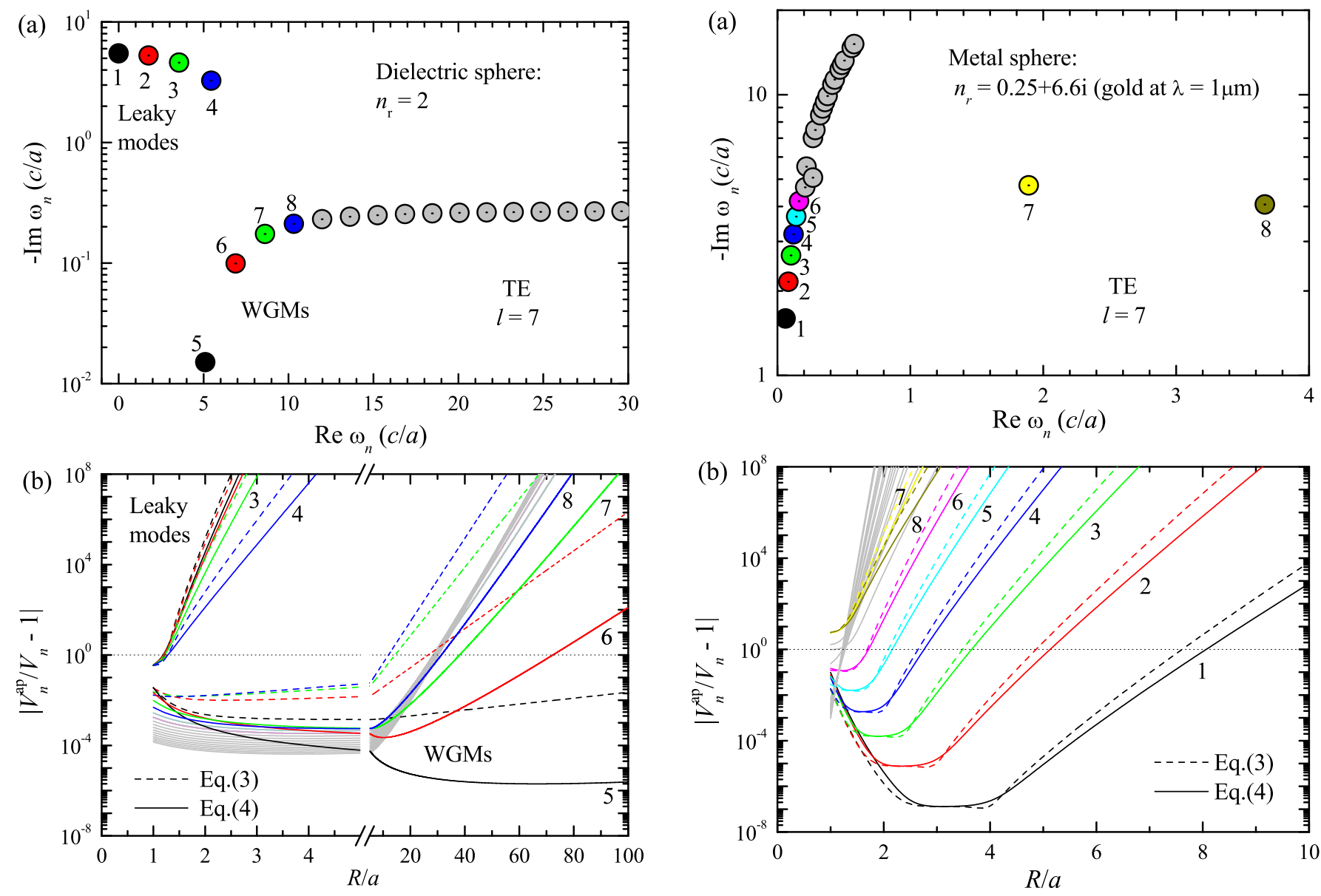

FIG. 3. (a) Frequencies of $l=7$ TE modes (RSs) of a dielectric sphere in vacuum, with permittivity $\epsilon=4$ and radius $a$. (b) Relative error of the approximate mode volume $\left|V_{n}^{\text {ap }} / V_{n}-1\right|$ as a function of the radius $R$ of the sphere of integration, for the modes shown in (a). $V_{n}^{\text {ap }}$ is calculated using Eq. (3), having no surface term, and Eq. (4), having the incorrect surface term used in the literature [4,5,7].

where $C_{l}(z)=(-1)^{l} i l(l+1)+O(1 / z)$. Similarly, for the normalization without the surface term, i.e., with the volume term only, Eq. (3) of the main text, we find

$$
\frac{V_{n}^{\mathrm{vol}}}{V_{n}}=I_{n}(R)=1+\frac{D_{l}\left(k_{n} R\right)}{\left(n_{r}^{2}-1\right)\left(k_{n} a\right)^{3} h_{l}^{2}\left(k_{n} a\right)} e^{2 i k_{n} R},
$$

where $D_{l}(z)=(-1)^{l} i+O\left(1 / z^{2}\right)$. Clearly, Eq. (4) brings an improvement compared to Eq. (3): the last term in Eq. (C15) is decreasing with $R$ for $Q \gg 1$ modes, such as WGMs, so that $\left|e^{2 i k_{n}(R-a)}\right| \approx 1$ up to rather large $R$. However both normalizations diverge for $R \rightarrow \infty$ due to the exponential factor $e^{2 i k_{n} R}$.

This is exemplified in Figs. 3(b) and 4(b) where the relative errors in the mode volume, $V_{n}^{\text {ap }} / V_{n}-1$ (with $V_{n}^{\text {ap }}=V_{n}^{\mathrm{LK}}$ or $\left.V_{n}^{\mathrm{ap}}=V_{n}^{\mathrm{vol}}\right)$, are shown for several RSs of a dielectric and a metal sphere, respectively, with corresponding eigenfrequencies given in Figs. 3(a) and 4(a). In Fig. 3(b), the strongest deviation and exponentially growing errors are seen for leaky modes already for small values of $R$. For WGMs the errors can be small up to rather large $R$, showing an apparent convergence, in agreement with the analytic treatment given

FIG. 4. As Fig. 3, but for a sphere with a fixed complex dielectric constant $\epsilon=-43.5+3.33 i$, equal to that of gold at a light wavelength of $\lambda=1 \mu \mathrm{m}$.

above, and the advantage of using Eq. (4) versus Eq. (3) is clearly observed. Nevertheless, the error diverges also for WGMs in the limit $R \rightarrow \infty$, in agreement with the asymptotics given by Eqs. (C15) and (C16). Moving to the metal sphere we observe that the modes have typically a low $Q_{n}$, such that the exponential divergence of the error with $R$ is more pronounced. For some low-frequency modes (labeled 1-5) the error initially decays exponentially up to a finite $R$ where the error is minimized. We note that this is observed both for Eq. (4) and Eq. (3), indicating that the surface term is not the relevant aspect here, and we find that it actually increases the error at small $R$. These states are quasibound states in the metal sphere which are evanescent close to the sphere due to the angular momentum, similar to WGMs. At $R \gtrsim l c /\left|\omega_{n}\right|$, they become propagating, and the error recovers the expected exponential divergence.

The correct normalization Eq. (8) can be analyzed in a similar way. It consists of two terms, $I_{n}(R)$ and $J_{n}(R)$, where

$$
\begin{aligned}
J_{n}(R) & =\frac{1}{2 k_{n}^{2}} \oint_{S_{R}} d S\left[\mathbf{E}_{n} \cdot \frac{\partial}{\partial r} r \frac{\partial \mathbf{E}_{n}}{\partial r}-r\left(\frac{\partial \mathbf{E}_{n}}{\partial r}\right)^{2}\right] \\
& =\frac{1}{2 k_{n}^{2}} \int d \Omega\left[\frac{1}{\sin ^{2} \theta}\left(\frac{\partial Y_{l m}}{\partial \varphi}\right)^{2}+\left(\frac{\partial Y_{l m}}{\partial \varphi}\right)^{2}\right]
\end{aligned}
$$




$$
\begin{aligned}
& \times A_{l}^{2} R^{2}\left[R_{l} \frac{\partial}{\partial r} r \frac{\partial R_{l}}{\partial r}-r\left(\frac{\partial R_{l}}{\partial r}\right)^{2}\right]_{r=R} \\
= & \frac{R^{3}}{a^{3}} \frac{z h_{l}(z) h_{l}^{\prime}(z)+z^{2} h_{l}(z) h_{l}^{\prime \prime}(z)-z^{2} h_{l}^{\prime 2}(z)}{\left(n_{r}^{2}-1\right) h_{l}^{2}\left(k_{n} a\right) z^{2}}
\end{aligned}
$$

with $z=k_{n} R$. We thus obtain

$$
I_{n}(R)+J_{n}(R)=1+\frac{R^{3}}{a^{3}\left(n_{r}^{2}-1\right)} \frac{p_{l}(z)}{h_{l}^{2}\left(k_{n} a\right) z^{2}},
$$

where

$$
\begin{aligned}
p_{l}(z)= & z h_{l}(z) h_{l}^{\prime}(z)+z^{2} h_{l}(z) h_{l}^{\prime \prime}(z)-z^{2} h_{l}^{\prime 2}(z) \\
& +z^{2} h_{l}^{2}(z)-z^{2} h_{l-1}(z) h_{l+1}(z)=0,
\end{aligned}
$$

according to Bessel's equation and recursive relations for Hankel functions [26] following from it. This confirms that Eq. (8) provides the exact normalization condition $I_{n}(R)+$ $J_{n}(R)=1$, independent of $R$.

\section{APPENDIX D: COMPARISON WITH SAUVAN ET $A L$.}

In the normalization introduced by Sauvan et al. [8] the electric field $\mathbf{E}$ of a RS (we drop here the index $n$ ) is normalized in such a way that

$$
I_{1}+I_{2}=1,
$$

where

$$
2 I_{1}=\int_{\mathcal{V}_{1}} d \mathbf{r} \mathbf{E} \cdot \frac{\partial(\omega \hat{\boldsymbol{\epsilon}}(\omega))}{\partial \omega} \mathbf{E}-\int_{\mathcal{V}_{1}} d \mathbf{r} \mathbf{H} \cdot \frac{\partial(\omega \hat{\boldsymbol{\mu}}(\omega))}{\partial \omega} \mathbf{H}
$$

is an integral over a volume $\mathcal{V}_{1}$ including the system inhomogeneity and $I_{2}$ is an integral of the same function over the region inside the PML surrounding $\mathcal{V}_{1}$, in which the field decays due to the artificial absorbing medium of the PML. Here $\mathbf{H}$ is the corresponding magnetic field of the RS. Note that we have used an extra factor of 2 in Eq. (D2), as compared to the equivalent equation in [8]. This is done in order to compare this normalization, which adds up contributions of the electric and magnetic field, with our normalization Eq. (B15), using the electric field only. We compare our normalization Eq. (B15) with Eq. (D1) by evaluating $I_{1}$, for which numerical values are provided in [8] for a TM mode of a gold sphere with radius $a=0.1 \mu \mathrm{m}$ having the wavelength $\lambda=2 \pi c / \omega=$ $(0.607+0.239 i) \mu \mathrm{m}$. We use the dielectric constant of gold in the Drude model with the same parameters as in $[8,27]$ : $\epsilon(\omega)=1-\lambda^{2} /\left[0.15^{2}(1+0.075 i \lambda)\right]$ with $\lambda$ measured in $\mu \mathrm{m}$.

The electric field of a TM mode has the form [13]

$$
\mathbf{E}(\mathbf{r})=\frac{A_{l}^{\mathrm{TM}}(k)}{\varepsilon(r) k r}\left(\begin{array}{c}
l(l+1) R_{l}(r, k) Y_{l m}(\Omega) \\
\frac{\partial}{\partial r} r R_{l}(r, k) \frac{\partial}{\partial \theta} Y_{l m}(\Omega) \\
\frac{\partial}{\partial r} \frac{r R_{l}(r, k)}{\sin \theta} \frac{\partial}{\partial \varphi} Y_{l m}(\Omega)
\end{array}\right)
$$

in which $k=\omega / c$ is a solution of the secular equation for TM modes

$$
\frac{1}{n_{r}} \frac{j_{l+1}\left(n_{r} k a\right)}{j_{l}\left(n_{r} k a\right)}=\frac{h_{l+1}(k a)}{h_{l}(k a)}-\frac{l+1}{k a}\left(1-\frac{1}{n_{r}^{2}}\right) ;
$$

$R_{l}(r, k)$ is given by Eq. (C7) and $\varepsilon(r)$ by Eq. (C5) with $n_{r}^{2}=$ $\epsilon(\omega)$, taking any of the two roots for $n_{r}$. The normalization constant $A_{l}^{\mathrm{TM}}(k)$ calculated using the correct normalization Eq. (B15) has the form

$$
\frac{n_{r} A_{l}}{A_{l}^{\mathrm{TM}}(k)}=\sqrt{\left[\frac{j_{l-1}\left(n_{r} k a\right)}{j_{l}\left(n_{r} k a\right)}-\frac{l}{n_{r} k a}\right]^{2}+\frac{l(l+1)}{k^{2} a^{2}}+\eta C_{l}(k),}
$$

where $A_{l}$ is given by Eq. (C8), and the last term under the square root takes into account the effect of the dispersion, with

$$
\eta=\frac{1}{\epsilon(\omega)} \frac{\partial\left(\omega^{2} \epsilon(\omega)\right)}{\partial\left(\omega^{2}\right)}-1
$$

and

$$
\left(n_{r}^{2}-1\right) C_{l}(k)=\frac{2(l+1)}{k^{2} a^{2}}+n_{r}^{2}\left[\frac{j_{l+1}^{2}\left(n_{r} k a\right)}{j_{l}^{2}\left(n_{r} k a\right)}-\frac{j_{l+2}\left(n_{r} k a\right)}{j_{l}\left(n_{r} k a\right)}\right] .
$$

Note that the normalization constant $A_{l}^{\mathrm{TM}}(k)$ of a TM mode, defined by Eq. (D5), generalizes the one used for a dielectric sphere, which is given by Eq. (29) of [13], as it takes into account the dispersion of the metal via the term $\eta C_{l}(k)$.

The corresponding magnetic field of the same RS has the form

$$
i \mathbf{H}(\mathbf{r})=A_{l}^{\mathrm{TM}}(k) R_{l}(r, k)\left(\begin{array}{c}
0 \\
\frac{1}{\sin \theta} \frac{\partial}{\partial \varphi} Y_{l m}(\Omega) \\
-\frac{\partial}{\partial \theta} Y_{l m}(\Omega)
\end{array}\right) .
$$

The integral $I_{1}=\left(I_{1 E}+I_{1 H}\right) / 2$ over a sphere of radius $R \geqslant a$ is then evaluated in the following way:

$$
\begin{aligned}
I_{1 E}= & \int_{\mathcal{V}_{R}} d \mathbf{r} \mathbf{E} \cdot \frac{\partial(\omega \hat{\boldsymbol{\epsilon}}(\omega))}{\partial \omega} \mathbf{E} \\
= & \frac{\left[A_{l}^{\mathrm{TM}}(k)\right]^{2}}{k^{2}}\left\{[l(l+1)]^{2} \int_{0}^{R} d r \frac{R_{l}^{2}(r, k) \beta(r)}{\varepsilon^{2}(r)}\right. \\
& \times \int d \Omega Y_{l m}^{2}(\Omega)+\int_{0}^{R} d r \frac{\beta(r)}{\varepsilon^{2}(r)}\left[\partial_{r} r R_{l}(r, k)\right]^{2} \\
& \left.\times \int d \Omega\left[\left(\partial_{\theta} Y_{l m}\right)^{2}+\frac{\left(\partial_{\varphi} Y_{l m}\right)^{2}}{\sin ^{2} \theta}\right]\right\} \\
= & \frac{\left[A_{l}^{\mathrm{TM}}(k)\right]^{2}}{k^{2}} l(l+1) \int_{0}^{R} d r \frac{\beta(r)}{\varepsilon^{2}(r)} \\
& \times\left[l(l+1) R_{l}^{2}+\left(\partial_{r} r R_{l}\right)^{2}\right],
\end{aligned}
$$

where

$$
\beta(r)=\left\{\begin{array}{cc}
\frac{\partial(\omega \epsilon(\omega))}{\partial \omega} & \text { for } r \leqslant a \\
1 & \text { for } r>a
\end{array}\right.
$$

and

$$
\begin{aligned}
I_{1 H} & =-\int_{\mathcal{V}_{R}} d \mathbf{r} \mathbf{H} \cdot \frac{\partial(\omega \hat{\boldsymbol{\mu}}(\omega))}{\partial \omega} \mathbf{H} \\
& =\left[A_{l}^{\mathrm{TM}}(k)\right]^{2} \int_{0}^{R} r^{2} R_{l}^{2} d r \int d \Omega\left[\left(\partial_{\theta} Y_{l m}\right)^{2}+\frac{\left(\partial_{\varphi} Y_{l m}\right)^{2}}{\sin ^{2} \theta}\right] \\
& =\left[A_{l}^{\mathrm{TM}}(k)\right]^{2} l(l+1) \int_{0}^{R} r^{2} R_{l}^{2} d r,
\end{aligned}
$$


TABLE I. The values of the integral Eq. (D2) calculated in the present work $\left(I_{1}\right)$ and the relative difference between $I_{1}$ and the value $I_{1}^{\mathrm{S}}$ calculated by Sauvan et al. [8], for three integration radii $R$, for the mode with the wavelength $2 \pi c / \omega=(0.607+0.239 i) \mu \mathrm{m}$ in a gold nanosphere of radius $a=0.1 \mu \mathrm{m}$.

\begin{tabular}{lccc}
\hline \hline$R(\mu \mathrm{m})$ & $I_{1}$ & $I_{1}^{\mathrm{S}} / I_{1}-1$ & $\left|I_{1}^{\mathrm{S}} / I_{1}-1\right|$ \\
\hline 0.15 & 0.61936187690 & $(5.66-1.29 i)$ & $5.80 \times 10^{-9}$ \\
& $-0.44899671324 i$ & $\times 10^{-9}$ & \\
1.0 & 6.56641919859 & $(0.057+2.095 i)$ & $2.095 \times 10^{-8}$ \\
& $+0.49127433385 i$ & $\times 10^{-8}$ & \\
2.0 & 1052.29778832465 & $(0.100+4.468 i)$ & $4.469 \times 10^{-8}$ \\
& $-1235.22683098918 i$ & $\times 10^{-8}$ & \\
\hline \hline
\end{tabular}

using $\hat{\boldsymbol{\mu}}=\hat{\mathbf{1}}$ everywhere. The integral in Eq. (D9) is calculated analytically using the Bessel equation and integration by parts:

$$
\begin{aligned}
& \int d r\left[l(l+1) R_{l}^{2}+\left(\partial_{r} r R_{l}\right)^{2}\right] \\
& \quad=r R_{l}^{2}+r^{2} R_{l} \partial_{r} R_{l}+\varepsilon k^{2} \int r^{2} R_{l}^{2} d r,
\end{aligned}
$$

where $\varepsilon$ is constant in each area of space. The integral in the last term of Eqs. (D11) and (D12) is a known analytic integral:

$$
\int x^{2} f_{l}^{2}(\alpha x) d x=\frac{x^{3}}{2}\left[f_{l}^{2}(\alpha x)-f_{l-1}(\alpha x) f_{l+1}(\alpha x)\right],
$$

in which $f_{l}(z)$ is any solution of the spherical Bessel equation, such as $j_{l}(z)$ or $h_{l}(z)$, and $\alpha$ is a complex constant. We therefore find

$$
\begin{aligned}
& I_{1 E}=\frac{\left[A_{l}^{\mathrm{TM}}(k)\right]^{2}}{k^{2}} l(l+1)\left(\frac{1}{\epsilon(\omega)} \frac{\partial(\omega \epsilon(\omega))}{\partial \omega} I_{1 E}^{a}+I_{1 E}^{R}\right), \\
& I_{1 H}=\frac{\left[A_{l}^{\mathrm{TM}}(k)\right]^{2}}{k^{2}} l(l+1)\left(I_{1 H}^{a}+I_{1 H}^{R}\right),
\end{aligned}
$$

where

$$
\begin{aligned}
I_{1 E}^{a}= & a(l+1)-\frac{n_{r} k a^{2}}{2}(2 l+3) \frac{j_{l+1}\left(n_{r} k a\right)}{j_{l}\left(n_{r} k a\right)} \\
& +\frac{n_{r}^{2} k^{2} a^{3}}{2}\left(1+\frac{j_{l+1}^{2}\left(n_{r} k a\right)}{j_{l}^{2}\left(n_{r} k a\right)}\right), \\
I_{1 E}^{R}= & \frac{1}{2 h_{l}^{2}(k a)}\left\{-k r^{2}(2 l+3) h_{l+1}(k r) h_{l}(k r)\right. \\
& \left.+2 r(l+1) h_{l}^{2}(k r)+k^{2} r^{3}\left[h_{l}^{2}(k r)+h_{l+1}^{2}(k r)\right]\right\}_{a}^{R}, \\
I_{1 H}^{a}= & {\left[\frac{k^{2} r^{3}}{2 j_{l}^{2}\left(n_{r} k a\right)}\left[j_{l}^{2}\left(n_{r} k r\right)-j_{l-1}\left(n_{r} k r\right) j_{l+1}\left(n_{r} k r\right)\right]\right]_{0}^{a}, } \\
I_{1 H}^{R}= & {\left[\frac{k^{2} r^{3}}{2 h_{l}^{2}(k a)}\left[h_{l}^{2}(k r)-h_{l-1}(k r) h_{l+1}(k r)\right]\right]_{a}^{R} . }
\end{aligned}
$$

The values of $I_{1}=\left(I_{1 E}+I_{1 H}\right) / 2$ calculated using Eqs. (D14) and (D15) are shown in Table I and compared with the values $I_{1}^{\mathrm{S}}$ provided by Sauvan et al. [8] for the same radii $R$ of the sphere of integration. One can see an excellent agreement between the two approaches, with a relative error in the $10^{-7}$ to $10^{-8}$ range. We note however that this result was obtained for a spherically symmetric system which is effectively 1D, and the actual calculation in [8] was done analytically [27], providing the excellent agreement with the strict result. In a full 3D calculation the use of a PML may lead to more significant errors. For instance, using the approach of [8], a deviation of about $2 \%$ of the PF from the direct numerical evaluation of the GF was found $[8,18]$ for an optical mode in a gold rod with cylindrical symmetry. It is also important to note that the derivation of the normalization in [8] uses a frequency-independent (nondispersive) PML, while in finite-difference time domain methods typically a dispersive PML is used to reduce numerical complexity. A more detailed comparison of this normalization method with Eq. (8) would be interesting.

\section{APPENDIX E: DETAILS OF THE PURCELL FACTOR CALCULATION}

In this section we provide some details of our calculation of the $\mathrm{PF}$ for a dielectric spherical resonator in vacuum, of radius $a$ and refractive index $n_{r}$; numerical results are presented here and in the main text. The PF is expressed in terms of the mode volumes via Eq. (9), and the mode volume of a RS is given by Eq. (2) of the main text, in terms of its normalized electric field. The latter has an explicit analytic form for a spherical resonator, which is given by Eq. (C6) for TE and by Eq. (D3) for TM polarization. The normalization constants are given by Eqs. (C8) and (D5), respectively. Static modes do not contribute to the PF as noted in Appendix A and thus are not considered here.

Owing to the spherical symmetry of the resonator, RS eigenfrequencies are $2 l+1$ degenerate with respect to the azimuthal quantum number $m$ (here $l$ is the orbital quantum number). Therefore, for each set of degenerate RSs, we introduce a collective mode volume $V_{l}$ defined as

$$
\frac{\mu^{2}}{V_{l}}=\sum_{m=-l}^{l}\left[\boldsymbol{\mu} \cdot \mathbf{E}_{l m}(\mathbf{r})\right]^{2},
$$

where the quantum numbers $l$ and $m$ are shown explicitly but the RS index $n$ is dropped for brevity of notation. Then, using the vector components of the dipole moment in spherical coordinates,

$$
\boldsymbol{\mu}=\mu_{r} \mathbf{e}_{r}+\mu_{\theta} \mathbf{e}_{\theta}+\mu_{\varphi} \mathbf{e}_{\varphi},
$$

and the sum rules for spherical harmonics,

$$
\sum_{m=-l}^{l}\left(\frac{1}{\sin \theta} \frac{\partial Y_{l m}}{\partial \varphi}\right)^{2}=\sum_{m=-l}^{l}\left(\frac{\partial Y_{l m}}{\partial \theta}\right)^{2}=l(l+1) \frac{2 l+1}{8 \pi}
$$

and

$$
\sum_{m=-l}^{l} Y_{l m}^{2}=\frac{2 l+1}{4 \pi}
$$

we obtain

$$
\begin{aligned}
\frac{\mu^{2}}{V_{l}^{\mathrm{TE}}} & =\frac{\left[E_{l}^{\mathrm{TE}}(r)\right]^{2}}{l(l+1)} \sum_{m=-l}^{l}\left[\mu_{\theta}^{2}\left(\frac{1}{\sin \theta} \frac{\partial Y_{l m}}{\partial \varphi}\right)^{2}+\mu_{\varphi}^{2}\left(\frac{\partial Y_{l m}}{\partial \theta}\right)^{2}\right] \\
& =\left[E_{l}^{\mathrm{TE}}(r)\right]^{2} \frac{2 l+1}{8 \pi}\left(\mu_{\theta}^{2}+\mu_{\varphi}^{2}\right)
\end{aligned}
$$


for TE modes, and

$$
\begin{aligned}
\frac{\mu^{2}}{V_{l}^{\mathrm{TM}}}= & {\left[E_{l}^{\mathrm{TM} 1}(r)\right]^{2} l(l+1) \sum_{m=-l}^{l} \mu_{r}^{2} Y_{l m}^{2}+\frac{\left[E_{l}^{\mathrm{TM} 2}(r)\right]^{2}}{l(l+1)} } \\
& \times \sum_{m=-l}^{l}\left[\mu_{\theta}^{2}\left(\frac{\partial Y_{l m}}{\partial \theta}\right)^{2}+\mu_{\varphi}^{2}\left(\frac{1}{\sin \theta} \frac{\partial Y_{l m}}{\partial \varphi}\right)^{2}\right] \\
= & {\left[E_{l}^{\mathrm{TM} 1}(r)\right]^{2} l(l+1) \frac{2 l+1}{4 \pi} \mu_{r}^{2} } \\
& +\left[E_{l}^{\mathrm{TM} 2}(r)\right]^{2} \frac{2 l+1}{8 \pi}\left(\mu_{\theta}^{2}+\mu_{\varphi}^{2}\right)
\end{aligned}
$$

for TM modes, where

$$
\begin{aligned}
E_{l}^{\mathrm{TE}}(r) & =\sqrt{\frac{2}{\left(n_{r}^{2}-1\right) a^{3}}} R_{l}(r), \\
E_{l}^{\mathrm{TM} 1}(r) & =\sqrt{\frac{2}{\left(n_{r}^{2}-1\right) a^{3} D_{l}}} \frac{1}{n_{r} k r} R_{l}(r), \\
E_{l}^{\mathrm{TM} 2}(r) & =\sqrt{\frac{2}{\left(n_{r}^{2}-1\right) a^{3} D_{l}}} \frac{1}{n_{r} k r} \frac{\partial}{\partial r} r R_{l}(r),
\end{aligned}
$$

$R_{l}(r)=j_{l}\left(n_{r} k r\right) / j_{l}\left(n_{r} k a\right), r$ is the position of the dipole (inside the dielectric sphere), and

$$
D_{l}=\left[\frac{j_{l-1}\left(n_{r} k a\right)}{j_{l}\left(n_{r} k a\right)}-\frac{l}{n_{r} k a}\right]^{2}+\frac{l(l+1)}{k^{2} a^{2}} .
$$

Note that the spherical harmonics $Y_{l m}$ used throughout this paper are taken in the form of real functions defined as [13]

$$
Y_{l m}(\theta, \varphi)=\sqrt{\frac{2 l+1}{2} \frac{(l-|m|) !}{(l+|m|) !}} P_{l}^{|m|}(\cos \theta) \chi_{m}(\varphi),
$$

where $P_{l}^{m}(x)$ are Legendre polynomials and

$$
\chi_{m}(\varphi)=\left\{\begin{array}{lll}
\pi^{-1 / 2} \sin (m \varphi) & \text { for } & m<0 \\
(2 \pi)^{-1 / 2} & \text { for } & m=0 \\
\pi^{-1 / 2} \cos (m \varphi) & \text { for } & m>0
\end{array}\right.
$$

respecting the orthonormality

$$
\int_{0}^{2 \pi} \chi_{m}(\varphi) \chi_{m^{\prime}}(\varphi) d \varphi=\delta_{m m^{\prime}},
$$

required by Eqs. (8) and (B28).

Equations (E7) and (E8) make $Y_{l m}(\theta, \varphi)$ different from their usual form adopted in the literature, in which exponentials $e^{i m \varphi}$ are used in the functions $\chi_{m}(\varphi)$. This exponential form suits the typical normalization in Hermitian systems, which uses a scalar product in a Hilbert space, with a complex conjugation of one field $\left(E E^{*}\right)$. For open systems, which are non-Hermitian, the proper normalization of RSs is given by Eq. (8). Using the exponential form, both integrals in Eq. (8) are vanishing, meaning that this form does not allow the RSs to be normalized. To understand this somewhat disconcerting finding, we consider a general combination of the degenerate pair of basis functions Eq. (E8) with $m= \pm|m|$ $(m \neq 0)$ :

$$
\tilde{\chi}_{m}(\varphi)=a_{m} \chi_{m}(\varphi)+b_{m} \chi_{-m}(\varphi),
$$

where $a_{m}$ and $b_{m}$ are constant coefficients. We require that the functions $\tilde{\chi}_{m}(\varphi)$ satisfy the same orthonormality condition Eq. (E9), from where we find that $a_{m}^{2}+b_{m}^{2}=1$ and $a_{m} b_{-m}+$ $a_{-m} b_{m}=0$. From these properties, simple algebra also yields the identities $a_{m}^{2}+b_{-m}^{2}=1$ and $a_{m} b_{m}+a_{-m} b_{-m}=0$. Inserting the resulting two degenerate RSs into Eq. (9), we find that, in accordance with Eqs. (E3) and (E4), their contribution to the Purcell factor, for the dipole component $\mu_{\varphi}$ in the TE and $\mu_{r}$ and $\mu_{\theta}$ in the TM polarization, is proportional to

$$
\begin{aligned}
\tilde{\chi}_{m}^{2}(\varphi)+\tilde{\chi}_{-m}^{2}(\varphi)= & \left(a_{m}^{2}+b_{-m}^{2}\right) \chi_{m}^{2}(\varphi)+\left(b_{m}^{2}+a_{-m}^{2}\right) \chi_{-m}^{2}(\varphi) \\
& +2\left(a_{m} b_{m}+a_{-m} b_{-m}\right) \chi_{m}(\varphi) \chi_{-m}(\varphi) \\
= & \chi_{m}^{2}(\varphi)+\chi_{-m}^{2}(\varphi)=\frac{1}{\pi}, \quad \text { (E11) }
\end{aligned}
$$

which is independent of the angle $\varphi$ and the choice of $a_{m}$ and $b_{m}$. Similarly, for the dipole component $\mu_{\theta}$ in the TE and $\mu_{\varphi}$ in the TM polarization, the Purcell factor is proportional to

$$
\left[\frac{d \tilde{\chi}_{m}}{d \varphi}\right]^{2}+\left[\frac{d \tilde{\chi}_{-m}}{d \varphi}\right]^{2}=\frac{m^{2}}{\pi},
$$

using the fact that $\chi_{m}^{\prime}(\varphi)=m \chi_{-m}(\varphi)$. We can clearly see that the orthonormality conditions make the PF independent of the basis choice, as must be expected for a physically relevant quantity.

To investigate further, we consider a transition to the exponential form along a parameter $\alpha$ choosing $a_{ \pm|m|}=$ $\pm \beta i \sin (\alpha)$ and $b_{ \pm|m|}=\beta \cos (\alpha)$. The orthogonality requires $\beta^{2}=1 / \cos (2 \alpha)$. The exponential form $e^{ \pm i|m| \varphi}$ corresponds to $\alpha=\pi / 4$, and we see that for this value of $\alpha$, the factor $\beta$ is diverging. The exponential form thus results in diverging amplitudes of the normalized RSs. This divergence is, however, exactly compensated by the above mentioned vanishing normalization integral for the modes taken in this form. The physical observable, the PF, is independent of basis choice, as shown by Eqs. (E11) and (E12), and remains valid also in the limit $\alpha \rightarrow \pi / 4$, owing to the cancellation of the divergence in Eq. (9).

The collective mode volumes of several RSs with $l=7$, calculated using Eqs. (E3)-(E5), are shown in Fig. 1(a) of the main text for TE polarization and in Figs. 5(a) and 6(a) for TM polarization and two different directions of the dipole. We note that the fundamental $n=1$ WGMs in TE and TM polarizations, which have quite similar $Q$ factors of the order of 100 , have very different mode volumes for a given direction of the dipole. Indeed, for an azimuthal dipole direction $\mathbf{e}=(0,0,1)$ the effective volume of the TE mode is much smaller than the one of the TM mode. This is because the electric field in TM polarization is mostly in radial direction, with only a small azimuthal component. For a radial direction of the dipole $\mathbf{e}=(1,0,0)$ instead, the TM mode has a much smaller mode volume, comparable to that of the TE mode for $\mathbf{e}=(0,0,1)$, as seen by comparing Figs. 1(a) and 6(a). The partial PFs due to all $l=7$ modes within the spectral range up to $\omega_{n} a / c \sim 40$ are shown separately, in Fig. 1(b) for TE and in Figs. 5(b) and 6(b) for TM polarization. These figures show the strong dependence of the PF on the dipole orientation, as discussed above. Summing over all different $l$ components and averaging over all possible directions of the dipole, we obtain the full PF for this system which is shown in Fig. 2 of the main text. 

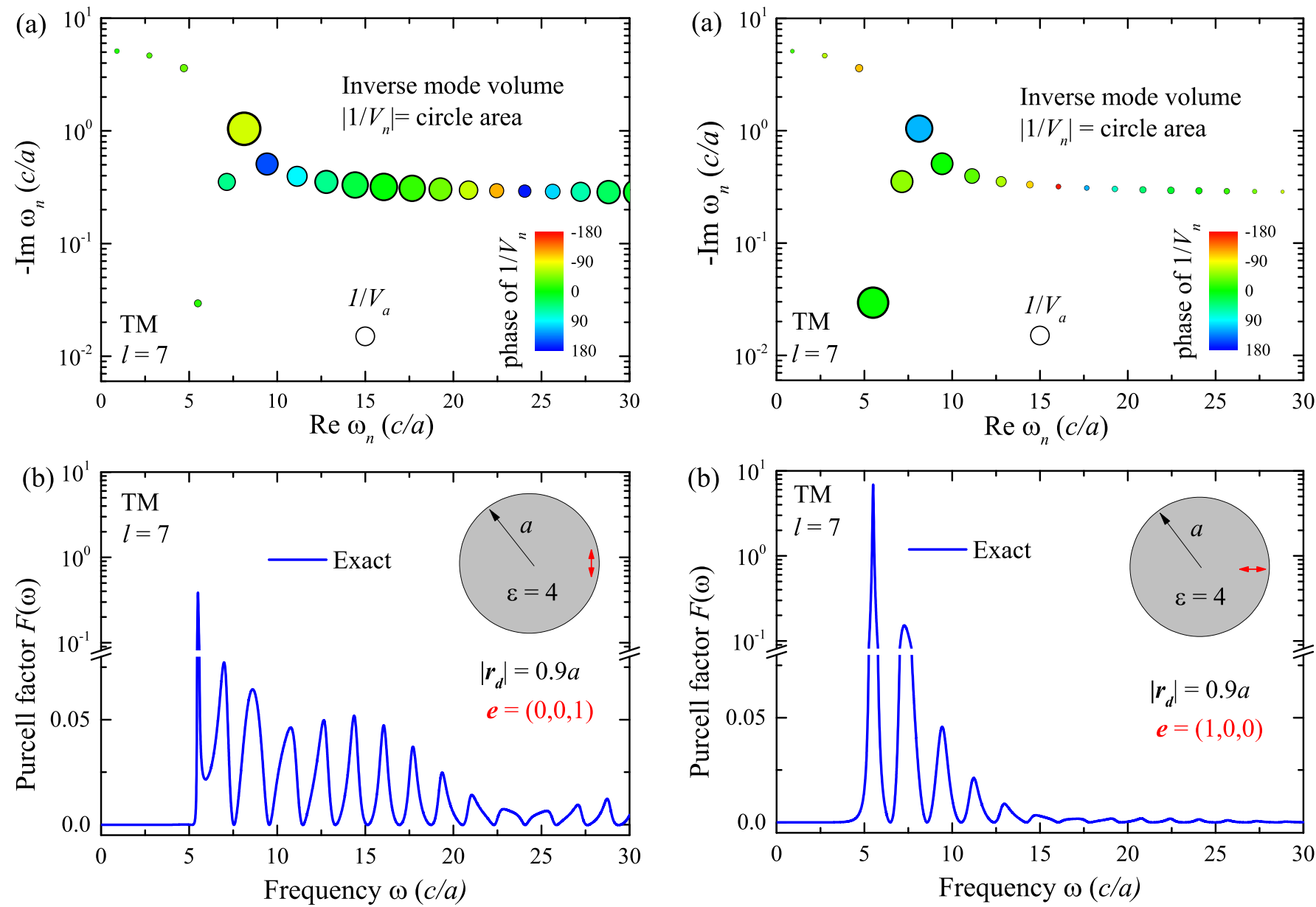

FIG. 5. (a) As Fig. 1(a) but for TM polarization. (b) Partial Purcell factor as a function of the dipole frequency $\omega$, calculated for the geometry of (a).

\section{APPENDIX F: VERIFICATION OF THE EXACT FORMULA FOR THE PURCELL FACTOR}

To verify the exact formula Eq. (9) for the PF we compare both the partial PF $F_{l}(\omega)$ for a given $l$, and the full PF $F(\omega)$ (i.e., the sum over all $l$ ) with those obtained via Eq. (5) using a direct evaluation of the analytic dyadic GF, resulting in $F_{l}^{\mathrm{a}}(\omega)$ and $F^{\text {a }}(\omega)$, respectively. The analytic form of the dyadic GF for a dielectric sphere in vacuum is known in the literature [28] and can be represented in terms of the linearly independent solutions of the second-order differential equation, given by spherical Bessel and Hankel functions. We use this analytic form both in TE and TM polarizations, for the same dielectric sphere and the same position of the dipole as considered in Figs. 1 and 5. We find excellent agreement, limited only by the finite number of RSs taken into account in the sums. As an example, we compare $F_{7}^{\text {a }}(\omega)$ with $F_{7}(\omega)$ in Fig. 7(a) and 7(b). The error $F_{7}^{\mathrm{a}}(\omega)-F_{7}(\omega)$ given in Fig. 7(c) shows that the analytic result is about $10^{-4}$ higher. This deviation is due to the limited number of RSs used to calculate $F_{7}$ via Eq. (9); we took into account all RSs with $\left|\omega_{n}\right|<\omega_{\max }=40 c / a$, as in the main text. The missing contributions of the higher frequency RSs yield an underestimation of the PF, which is increasing with frequency as one approaches the frequencies of the missing RSs.

FIG. 6. As Fig. 5 but for an orthogonal direction of the dipole: $\mathbf{e}=(1,0,0)$.

Summing over all partial PFs with $l<\omega_{\max } a / c$, we obtain the full PF, both in TE and TM polarizations for the same dipole; see Figs. 8(a) and 8(b). Its error $F^{\mathrm{a}}(\omega)-F(\omega)$ shown in Fig. 8(c) is again positive and slightly increasing with frequency. It is larger than the partial error shown in Fig. 8(c) due to the accumulated errors from different $l$. These errors are oscillating and decaying in magnitude with increasing cutoff frequency $\omega_{\max }$, as shown in the inset of Fig. 8(c). A convergence faster than $\omega_{\max }^{-1}$ is observed, as indicated by the dotted lines.

\section{APPENDIX G: APPLICATION OF EXACT NORMALIZATION TO MODES CALCULATED WITH NUMERICAL SOLVERS}

We have shown in Figs. 3 and 4 the LK normalization for a finite radius $R$, having sizable errors close to the system (of around $10 \%$ for the examples shown), and diverging errors in the limit of infinite $R$. We can see that, when excluding the leaky modes which have $Q_{n}<3$, the divergence for large radii only becomes apparent for $R / a>10$, a regime that in a numerical simulation would not likely be explored due to the large required computational domain. The errors at small radii are therefore typically the more significant limitation of the LK normalization for simulations of high- $Q$ modes. 


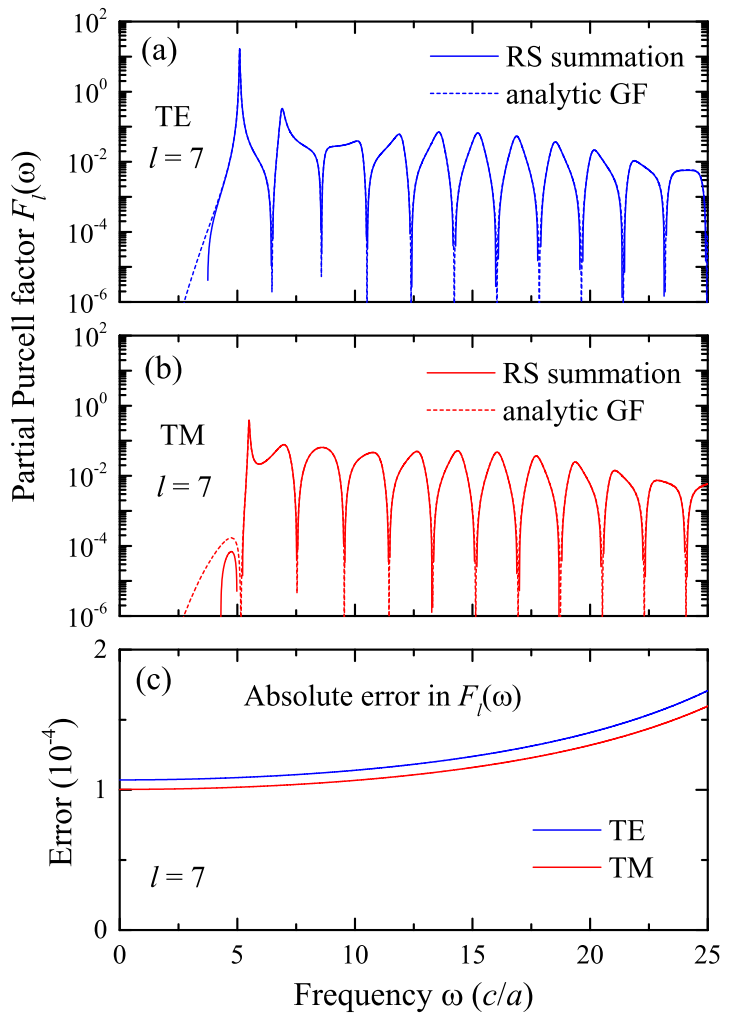

FIG. 7. (a) Partial Purcell factor of a dielectric sphere in vacuum, with permittivity $\varepsilon=4$ and radius $a$, for a point dipole placed at $\left|\mathbf{r}_{d}\right|=0.9 a$ with direction $\mathbf{e}=(0,0,1)$, calculated for TE polarization and $l=7$ via the RSs summation Eq. (9) $\left[F_{7}(\omega)\right.$, solid line] and by using the analytic form of the dyadic GF $\left[F_{7}^{\mathrm{a}}(\omega)\right.$, dotted line]. (b) As (a) but for TM polarization. (c) Errors $F_{7}^{\mathrm{a}}(\omega)-F_{7}(\omega)$ for TE and TM polarization using a summation cutoff $\omega_{\max }=40$.

It is worthwhile at this point to give an intuitive picture of the difference between the LK and the exact normalization, which we mentioned in the main text. If we consider a plane wave propagating with wave vector $\mathbf{k}$ in vacuum, we can write its phase as $\mathbf{k} \cdot \mathbf{r}=r(\omega / c) \cos \alpha$, where $r$ is the magnitude of $\mathbf{r}$ and $\alpha$ is the angle between the propagation direction and the direction of $\mathbf{r}$. Evaluating the surface term of the normalization on a small part of a spherical surface, we obtain that the surface term in Eq. (8) is a factor $\cos \alpha$ smaller than in Eq. (4). This gives us a physical picture of the difference between the two normalizations. The LK normalization assumes a propagation normal to the surface of integration, while the exact normalization takes into account the actual propagation direction. To determine the actual propagation direction, spatial derivatives of the fields are required, and are therefore present in Eq. (8).

We now can also understand why the LK normalization would be valid for $s$ waves and a spherical surface around the center; in this case the propagation direction would be radial. However, pure $s$ waves (such as $l=0$ modes of a sphere) do not exist in electrodynamics of finite systems, as already mentioned in Appendix C. We note that Kristensen et al. have recently published an article [29] in which they show in Figs. 2 and 3 the LK normalization for a geometry with an effectively $s$-wave mode, using a two-dimensional problem with trans-
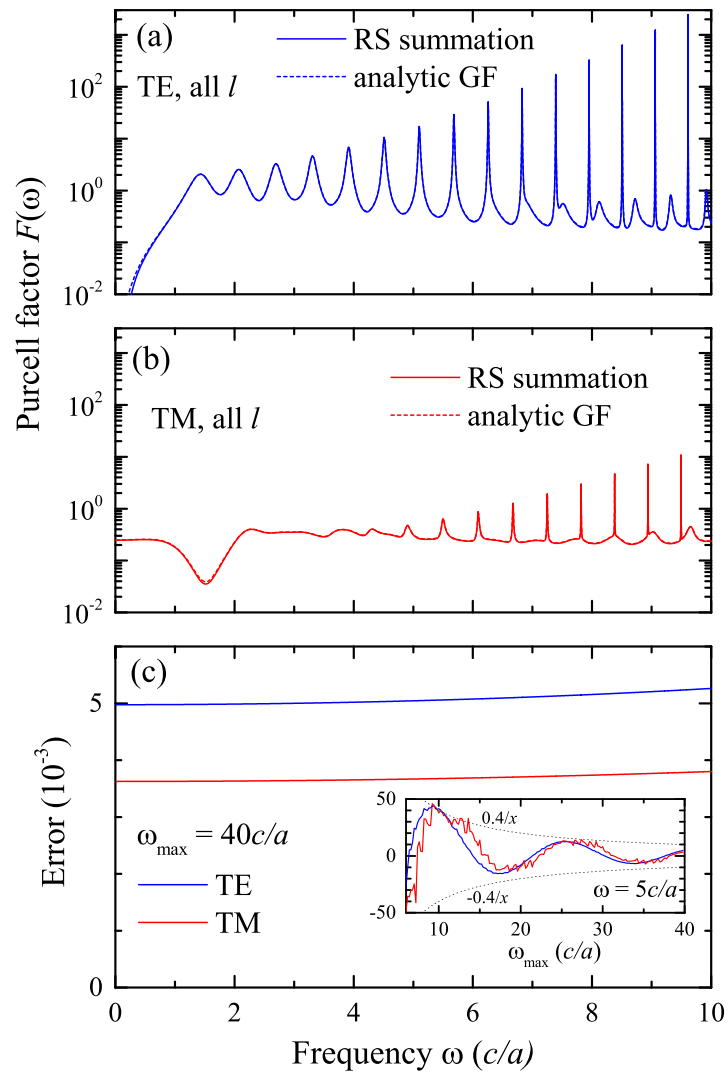

FIG. 8. (a) Full Purcell factor of a dielectric sphere in vacuum, with permittivity $\varepsilon=4$ and radius $a$, for a point dipole placed at $\left|\mathbf{r}_{d}\right|=$ $0.9 a$ with direction $\mathbf{e}=(0,0,1)$, calculated for TE polarization via the RSs summation Eq. (9) $[F(\omega)$, solid line] and by using the analytic form of the dyadic GF [ $F^{\mathrm{a}}(\omega)$, dotted line]. (b) As (a) but for TM polarization. (c) Errors $F^{\mathrm{a}}(\omega)-F(\omega)$ for TE and TM polarization using a summation cutoff $\omega_{\max }=40 c / a$. The inset shows the errors at $\omega=5 c / a$ as function of $\omega_{\max }$, with the convergence illustrated by the dotted lines showing $\pm 0.4 c /\left(\omega_{\max } a\right)$.

lation invariance along $z$ and electric field along $z$. This is a well chosen example to show that the LK normalization can have small errors. The second example shown is an axially symmetric geometry of a gold nanorod dimer, and a dipolar mode is shown having the emitted field dominantly polarized along the axial direction and thus again a dominant $s$ character, leading to reduced errors of the LK normalization.

Using this insight we can understand the resulting errors of the LK normalization. At small distances from the system, the propagation direction will significantly differ from being radial, simply due to the geometrical factor. This is the reason for the significant errors at small distances seen in Figs. 3 and 4 . Going to large distances, the angle $2 \alpha$, at which the system is seen from the distance, will scale as $1 / R$, so that the error, $1-\cos \alpha$, scales as $1 / R^{2}$. Note that this is consistent with the analytical result for the Mie modes in Eq. (C15) and also in Eq. (26) of [29]. For resonant states with finite loss, this leads to the divergence at large distances $\propto \exp \left(2 i \omega_{n} R / c\right) / R^{2}$, where the exponential increase of the field dominates the $R^{-2}$ decay in the LK normalization error.

Importantly, this discussion shows that even if we can neglect the error due to the exponential divergence, for 
example by considering high- $Q$ modes, the error of the surface term in Eq. (4) is still approximately $L^{2} /(2 R)^{2}$, where $L$ is the system size. In order to provide an error of $1 \%$, which could be considered sufficient, one still needs a simulation domain which is about five times the system size. Simulating such large areas is computationally costly, specifically in three dimensions, where a simulation volume of about 100 times the system volume would be required. It is also important to note that the LK normalization requires a spherical normalization volume with the system in the center in order to provide the $R^{-2}$ scaling of the propagation direction error. For other volume shapes it is not converging to the correct normalization even for modes of infinite $Q_{n}$.

The exact normalization instead can be used for any volume enclosing the system, with arbitrary volume shapes. Therefore the exact normalization is also advantageous for numerical evaluation, as it does not require an extended or specifically shaped simulation domain.

In order to exemplify this discussion and explicitly show the applicability of the exact normalization to modes determined with numerical solvers, we have calculated modes of a dielectric cylinder using the FEM solver COMSOL. The cylinder has a height $h$ equal to its diameter $2 a$, and a refractive index of 3 . We used the axisymmetric eigenmode solver of COMSOL, and an angular quantum number of $m=1$. The cylinder is embedded in vacuum, and the simulation area is enclosed by a spherical PML at a radius of $5 \lambda+2 \sqrt{2} a$, where $\lambda$ is the target vacuum mode wavelength $\lambda=2 a$. The PML thickness is $\lambda$. The mesh was determined by a single mesh parameter $\Delta_{\mathrm{m}}$, from which the mesh was created using a free triangular mesh with a minimum size of $\Delta_{\mathrm{m}} / 3$ in the cylinder, $\Delta_{\mathrm{m}}$ in vacuum and the PML, and a maximum size twice the minimum size in all regions. It is important to note that, in order to show the $R$ dependence of the normalization, we used a large simulation domain, which leads to large numerical complexity. Furthermore, spurious surface (whispering gallery) modes, forming at the vacuum to PML interface due to the nonideality of the PML, constitute most of the modes found by the eigenmode solver of COMSOL, when using such a large simulation domain, which makes it cumbersome to find the nonspurious modes of the system.

To evaluate the normalization integrals, we exported the mode field into a square grid of $\Delta_{\mathrm{g}}$ pitch. We calculated the first and second derivatives using a three-point differential scheme with an error scaling as $O\left(\Delta_{\mathrm{g}}^{2}\right)$. The surface integrals were evaluated by dividing the half circle in cylindrical coordinates representing the surface by 1025 linear segments [20].

We show here the results for a mode with a frequency of $\omega_{n} a / c=2.943508477-0.1724037812 i$ having a $Q_{n}$ of about 8.5. For illustration, the electric field distribution in the cylindrical coordinate plane $(\rho, z)$ is shown in Fig. 9(c). The far-field emission pattern has three nodes showing its non- $s$-wave character. The effect of the exponential divergence of the mode towards larger radius is evident, as the $R^{-1}$ decay due to the three-dimensional emission is superseded by the exponential growth of $\left|\exp \left(i \omega_{n} R / c\right)\right|$.

To determine the normalization we evaluate Eq. (8) as function of $R$. We find numerical noise which increases with increasing $R$, proportional to the surface term. We therefore choose as mode normalization $V_{n}$ the value for which the
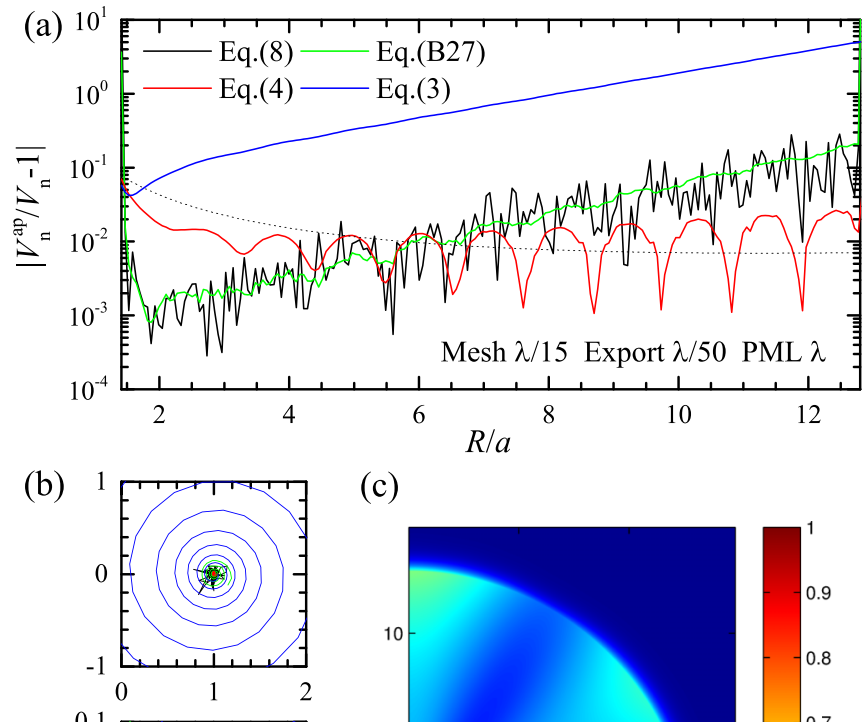

(c)
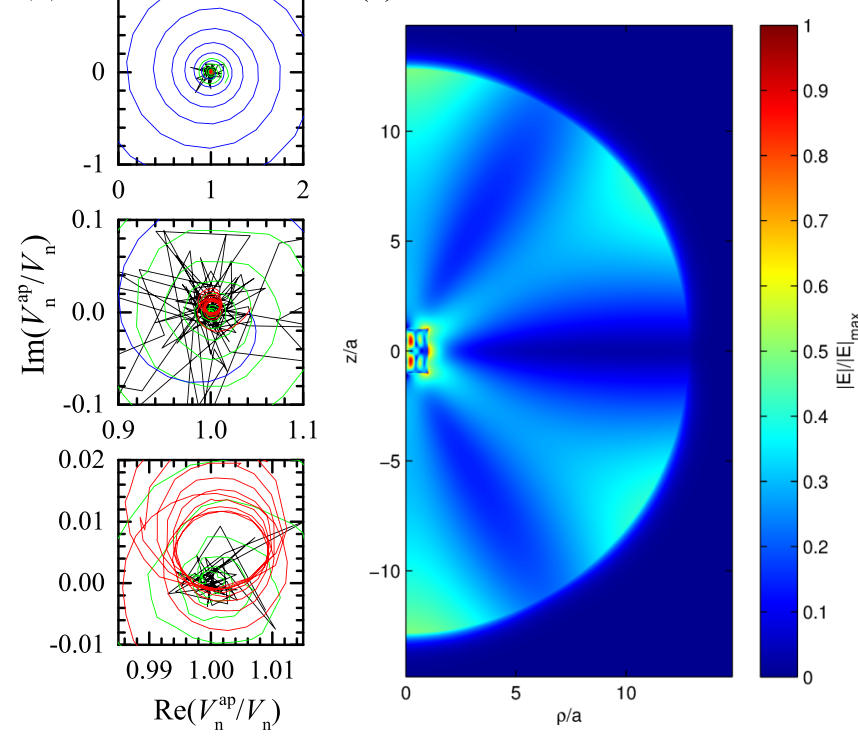

FIG. 9. Normalization of a RS of angular quantum number $m=1$ of a cylinder of radius $a$ and height $2 a$, calculated using COMSOL, using $\Delta_{\mathrm{m}}=\lambda / 15$, and an export mesh $\Delta_{\mathrm{g}}=\lambda / 50$. (a) Relative error of the approximate mode volume $\left|V_{n}^{\text {ap }} / V_{n}-1\right|$ as function of the radius $R$ of the sphere of integration. $V_{n}^{\text {ap }}$ is calculated without surface term Eq. (3), or the LK surface term Eq. (4). Additionally the results of the correct surface term using first and second derivatives Eq. (8) and only first derivatives Eq. (B27). The dashed line indicates a term $\propto$ $\left|\exp \left(2 i \omega_{n} R / c\right) / R^{2}\right|$. (b) Relative complex approximate mode volume $V_{n}^{\text {ap }} / V_{n}$ for $R / a$ varying from 1.5 to 12.3 . Three different zooms are shown. Calculated points are connected by straight lines. For the highest zoom the results of Eq. (8) are shown only for $R / a<5$. (c) Mode field amplitude $\left|E_{n}(\mathbf{r})\right|$ in cylindrical coordinates $(\rho, z)$.

surface integral is small, at about $R / a=2$. The resulting $R$-dependent normalization errors are given in Fig. 9(a). Let us first discuss the result of Eq. (8). We can see a value which is randomly fluctuating around zero [see also the complex normalized mode volume in Fig. 9(b)], showing that the deviation is due to numerical errors. Comparing this error with the error of the volume-only normalization Eq. (3), we see that it is about 1-2 orders of magnitude smaller, indicating that the relative error of the surface integral evaluation is about $1 \%-10 \%$ in this case. Interestingly, using the first-derivative expression for the exact normalization, Eq. (B27), this random error is converted into a systematic error of similar magnitude, but spiraling in the complex plane. We have found that choosing different $\Delta_{\mathrm{g}}$ changes these numerical errors, and 


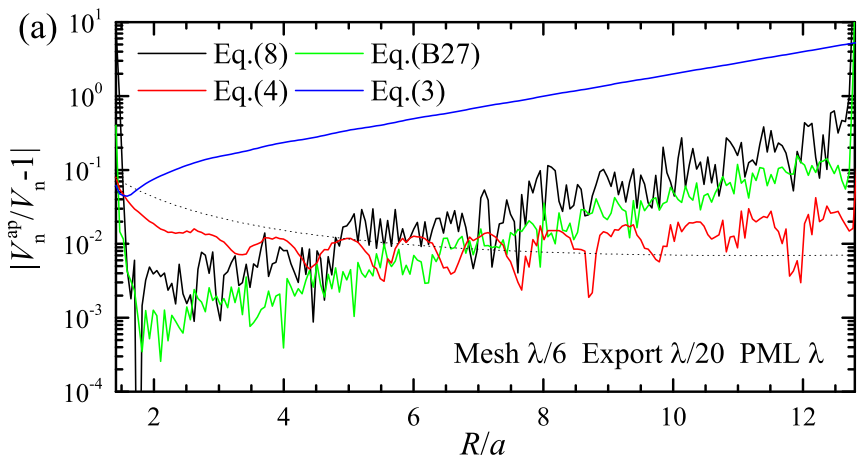

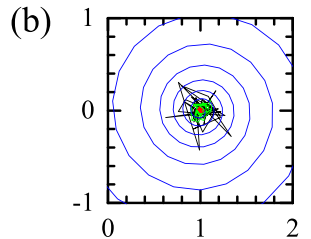
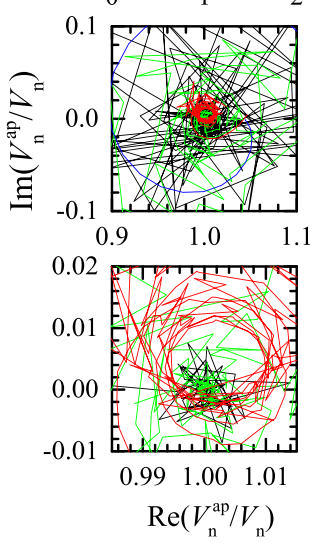

(c)

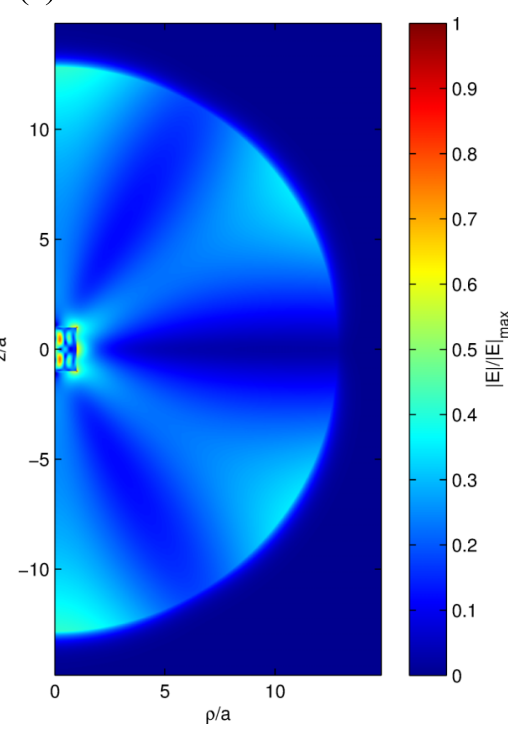

FIG. 10. As Fig. 9, but using a mesh of $\lambda / 6$, and an export mesh $\lambda / 20$.

we have chosen the ratio between $\Delta_{\mathrm{g}}$ and $\Delta_{\mathrm{m}}$ to provide the smallest errors within a factor of two for both exact normalization formulations. We found that for Eq. (B27) the optimum $\Delta_{\mathrm{g}}$ was about three times smaller than for Eq. (8).

The error of the correct surface integral is dominated by the error in determining the local propagation direction, i.e., by the spatial derivatives of the fields. To minimize the resulting error of the normalization, it is therefore best to evaluate the normalization using a volume with a small surface term, as we have done. Too close to the system, strong field gradients and spatial variations of the mesh can give rise to additional numerical errors, as visible in Fig. 9(a) for $R<2 a$.

We find the error of Eq. (4) to be significant for small $R$, similar to what is observed for the Mie modes in Fig. 3 and explained as the failure of the radial propagation assumption of Eq. (4). With increasing $R$ the value oscillates, due to a rotation of the error in the complex plane as shown in Fig. 9(b), according to the scaling $\exp \left(2 i \omega_{n} R / c\right) / R^{2}$ shown by the dashed line in Fig. 9(a). Additional to the oscillation, we also observe a slow drift of the center position, which we discuss later on.

We now compare these result with the ones for a 2.5 times coarser mesh $\left(\Delta_{\mathrm{m}}=\lambda / 6\right.$ and $\left.\Delta_{\mathrm{g}}=\lambda / 20\right)$ shown in Fig. 10, which leads to a very small relative change of $\omega_{n}$ of $-1.2 \times 10^{-6}$ for the real part and $-7.8 \times 10^{-5}$ for the

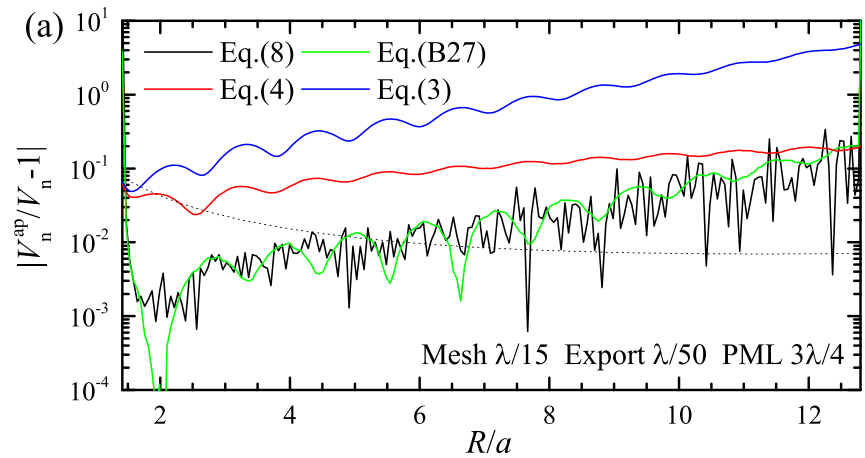

(b)
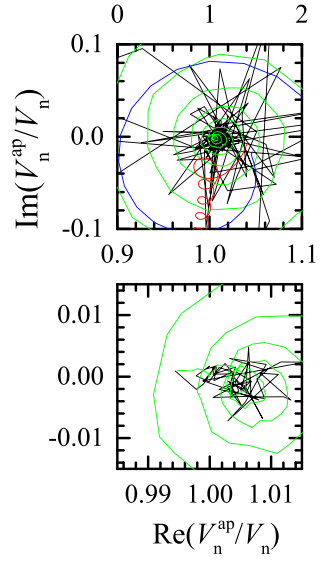

(c)

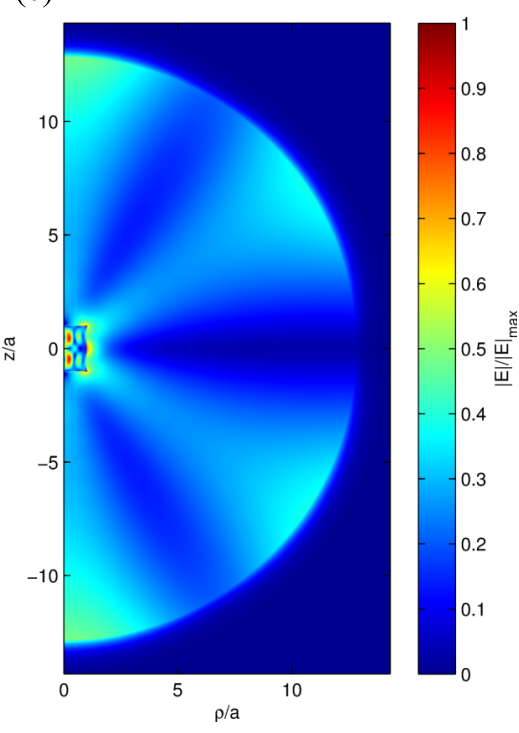

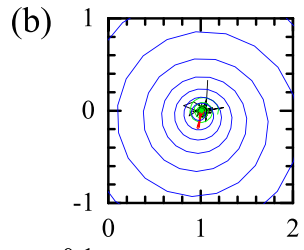

FIG. 11. As Fig. 9, but using a PML thickness of $3 \lambda / 4$.

imaginary part. This mesh size is advised in typical COMSOL examples. We find that the results are generally similar, but show higher random numerical errors. Notably, we find that the first-derivative formulation Eq. (B27) shows now an error about five times lower than the one of the second-derivative formulation Eq. (8), and having a magnitude similar to that for the finer mesh shown before. This illustrates an advantage of the first-derivative normalization for coarser grid calculations having larger numerical errors.

We now look at the effects of the nonideality of the PML, producing reflected waves. Such waves are incoming, and thus have the opposite propagation direction to the one assumed in Eq. (4). We used the fine mesh of Fig. 9, but a PML thickness reduced from $\lambda$ to $3 \lambda / 4$. This leads to a relative change of $\omega_{n}$ of $-3.5 \times 10^{-3}$ for the real part and $-8.2 \times 10^{-3}$ for the imaginary part. While the presence of the reflected wave is hardly visible in the mode amplitude shown in Fig. 11(c), the error of Eq. (3) in Fig. 11(a) is clearly showing oscillations due to the interference of outgoing and incoming waves. Their contrast is increasing with decreasing $R$ due to the exponential growth of the reflected incoming field with decreasing $R$, similar to the growth with increasing $R$ of the outgoing field. Evaluating the total field growth due to $\operatorname{Im}\left(\omega_{n}\right)$ during the propagation to the PML and back to the center we find a factor of about 15 for the present 

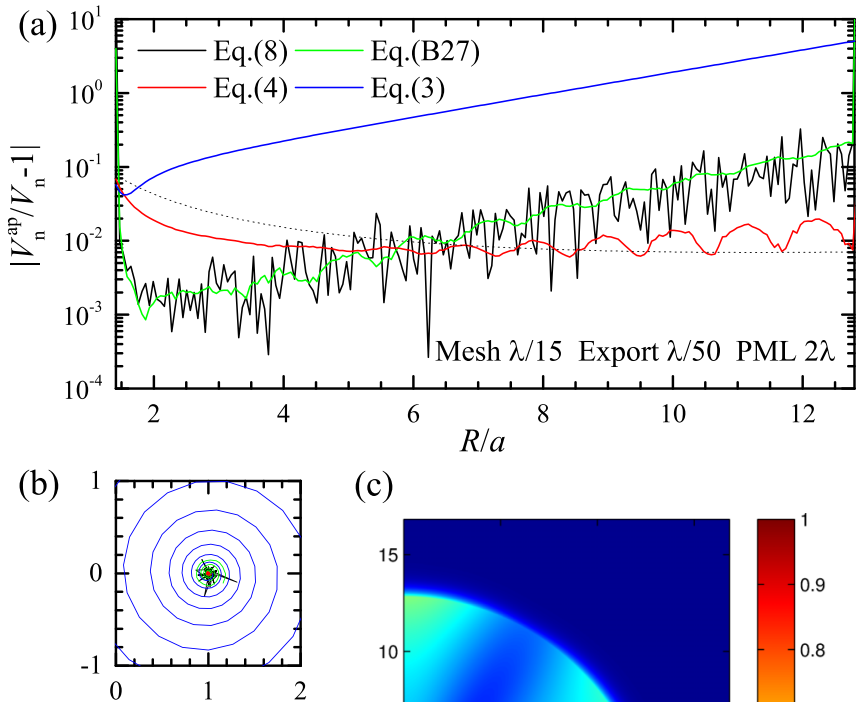

(c)
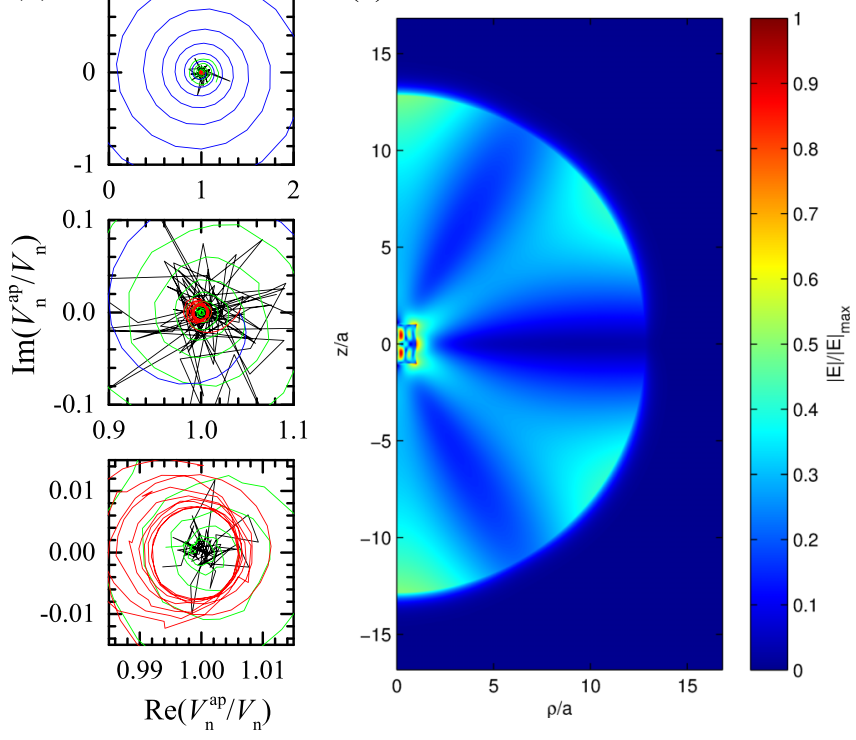

FIG. 12. As Fig. 9, but using a PML thickness of $2 \lambda$.

case. Using large simulation volumes as done here results in such large factors, which give rise to artifacts even for a low reflectivity of the PML. Again for the present case, we see a contrast of about $20 \%$ close to the center, corresponding to the incoming field amplitude of about $10 \%$ of the outgoing one, and thus to a PML amplitude reflectivity of about $0.7 \%$, or intensity reflectivity of about $5 \times 10^{-5}$. Very small residual reflectivities of the PML can thus lead to large contributions of incoming waves, specifically for modes of low $Q_{n}$.

Looking at the error of the LK normalization in Fig. 11(a), we see the significant impact of the incoming waves. The behavior in the complex plane in Fig. 11(b) is instructive; in addition to the spiraling, the normalization is drifting in phase approximately linearly with $R$ by up to 0.2 radians.

The drift of the LK normalization seen in Fig. 9 is therefore attributed to a small reflection of the PML, which can be also seen as weak oscillation of the volume normalization. Increasing the thickness of the PML to $2 \lambda$, shown in Fig. 12, this drift is reduced, and the center of rotation of the LK normalization is found closer to the exact normalization. The relative change of $\omega_{n}$ due to this increased PML thickness is $2.1 \times 10^{-4}$ for the real part and $6 \times 10^{-4}$ for the imaginary part.

Interestingly, the exact normalization is hardly influenced by such reflections, which is understandable since it takes into account the propagation direction. To compare the normalization of the modes for the different simulations, we use the volume integral Eq. (3) evaluated for the smallest possible radius of the sphere fully including the cylinder, $R=\sqrt{2} a$. This integral is proportional to the electric field squared and consequently to the inverse mode volume. For the exact normalization of the mode, we find the value of the volume integral to be $1.05735-0.00126 i$ for Fig. 9, $1.06158-0.00108 i$ for Fig. 10, $1.06469-0.01038 i$ for Fig. 11, and $1.05704-0.00202 i$ for Fig. 12. This shows that the exact normalization is stable to $0.7 \%$ for all these cases. At the same time the LK normalization has minimum errors above $4 \%$ in the case of Fig. 11 .

To summarize, we have presented an example of the applicability of the exact normalization to numerically determined modes, which indicates the following. First, as the exact normalization can be evaluated over any volume containing the system, this volume can be chosen close to the system to have a small surface term and therefore smaller errors, and not requiring an extension of the simulation domain. Second, the fact that the exact normalization takes proper account of the propagation direction makes it less susceptible to incoming waves, such as those propagating from nonideal PMLs, so that the exact normalization is robust against such errors in numerical simulations.

Considering the LK normalization instead, we emphasize that it assumes that the field at the surface is propagating normal to the surface of the normalization volume. It therefore does not determine the propagation direction from the field gradients, and is consequently having smaller numerical errors. However, this assumption creates systematic errors which depend on the specific mode analyzed and the surface geometry used. For the widely used spherical volume, the resulting error scales $\propto \exp \left(2 i \omega_{n} R / c\right) / R^{2}$, where the $1 / R^{2}$ factor simply comes from the angular size of the system seen from the surface of integration. If the mode frequency $\omega_{n}$ is real, the error is converging to zero at $R \rightarrow \infty$. For any lossy mode, the finite imaginary part of $\omega_{n}$ leads to a divergence of the error at $R \rightarrow \infty$. For modes with a sufficiently large $Q$ factor, this divergence however is seen only for $R$ larger than any numerically treatable domains. Even in this case, a significant reduction of the errors due to non-normal propagation requires large values of $R$, as shown in Figs. 3 and 9-12. Therefore in practical terms, the most problematic feature of the LK normalization for high- $Q$ modes is not the divergence at $R \rightarrow \infty$, but the requirement to use simulation sizes much larger than the system size, in order to approximately achieve the normal incidence condition.
[1] E. M. Purcell, Phys. Rev. 69, 681 (1946).

[2] R. Coccioli et al., IEE Proc.-Optoelectron. 145, 391 (1998).
[3] For consistency with results of the present paper, we have removed from the original formula the factor of $\varepsilon\left(\mathbf{r}_{d}\right)$ and added the dipole polarization vector $\mathbf{e}$. 
[4] P. Kristensen, C. van Vlack, and S. Hughes, Opt. Lett. 37, 1649 (2012).

[5] P. T. Kristensen and S. Hughes, ACS Photonics 1, 2 (2014).

[6] P. T. Leung, S. Y. Liu, and K. Young, Phys. Rev. A 49, 3057 (1994).

[7] P. T. Leung and K. M. Pang, J. Opt. Soc. Am. B 13, 805 (1996).

[8] C. Sauvan, J. P. Hugonin, I. S. Maksymov, and P. Lalanne, Phys. Rev. Lett. 110, 237401 (2013).

[9] E. A. Muljarov, W. Langbein, and R. Zimmermann, Europhys. Lett. 92, 50010 (2010).

[10] M. B. Doost, W. Langbein, and E. A. Muljarov, Phys. Rev. A 85, 023835 (2012).

[11] M. B. Doost, W. Langbein, and E. A. Muljarov, Phys. Rev. A 87, 043827 (2013).

[12] L. J. Armitage, M. B. Doost, W. Langbein, and E. A. Muljarov, Phys. Rev. A 89, 053832 (2014).

[13] M. B. Doost, W. Langbein, and E. A. Muljarov, Phys. Rev. A 90, 013834 (2014).

[14] E. A. Muljarov and W. Langbein, Phys. Rev. B 93, 075417 (2016).

[15] R. J. Glauber and M. Lewenstein, Phys. Rev. A 43, 467 (1991).
[16] H. T. Dung, L. Knöll, and D.-G. Welsch, Phys. Rev. A 62, 053804 (2000).

[17] H. T. Dung, L. Knöll, and D.-G. Welsch, Phys. Rev. A 64, 013804 (2001).

[18] Q. Bai et al., Opt. Express 21, 27371 (2013).

[19] H. Levine and J. Schwinger, Commun. Pure Appl. Math. 3, 355 (1950).

[20] The data presented in this work are available from the Cardiff University data archive, http://doi.org/10.17035/ d.2016.0012064868.

[21] G. D. Mahan, Many-Particle Physics (Plenum, New York, 1990).

[22] O. J. F. Martin and N. B. Piller, Phys. Rev. E 58, 3909 (1998).

[23] R. M. More, Phys. Rev. A 4, 1782 (1971).

[24] J. Bang and F. A. Gareev, Lett. Nuovo Cimento 32, 420 (1981).

[25] M. Born and E. Wolf, Principles of Optics, 7th ed. (Cambridge University Press, 1999), p. 423.

[26] M. Abramowitz and I. A. Stegun, Handbook of Mathematical Functions (Dover, New York, 1965).

[27] C. Sauvan (private communication).

[28] W. Chew, Waves and Fields in Inhomogeneous Media (IEEE Press, Piscataway, NJ, 1995).

[29] P. T. Kristensen, R.-C. Ge, and S. Hughes, Phys. Rev. A 92, 053810 (2015). 